\title{
Stochastic approximation for risk-aware Markov decision processes
}

\author{
Wenjie Huang * $\quad$ William B. Haskell ${ }^{\dagger}$
}

December 5, 2019

\begin{abstract}
We develop a stochastic approximation-type algorithm to solve finite state/action, infinite-horizon, risk-aware Markov decision processes. Our algorithm has two loops. The inner loop computes the risk by solving a stochastic saddle-point problem. The outer loop performs $Q$-learning to compute an optimal risk-aware policy. Several widely investigated risk measures (e.g. conditional value-at-risk, optimized certainty equivalent, and absolute semi-deviation) are covered by our algorithm. Almost sure convergence and the convergence rate of the algorithm are established. For an error tolerance $\epsilon>0$ for the optimal $Q$-value estimation gap and learning rate $k \in(1 / 2,1]$, the overall convergence rate of our algorithm is $\Omega\left(\left(\ln (1 / \delta \epsilon) / \epsilon^{2}\right)^{1 / k}+(\ln (1 / \epsilon))^{1 /(1-k)}\right)$ with probability at least $1-\delta$.
\end{abstract}

Keywords: Markov decision processes; Risk measure; Saddle-point; Stochastic approximation; $Q$-learning.

\section{Introduction}

The analysis of complex systems such as inventory control, financial markets, waste-to-energy plants, and computer networks is difficult because of the intrinsic uncertainty in these systems. Risk-aware optimization offers a possible remedy by searching for strong reliability guarantees. In particular, it gives more attention to low probability but high cost events than a risk-neutral optimizer would. Risk awareness is especially important in sequential decision-making.

Markov decision processes (MDPs) introduced by Bellman in [10] provide a mathematical framework for sequential decision-making. However, the exact model of the underlying MDP is often unknown and one can only observe the trajectory of states, actions, and costs. $Q$-learning, as developed in [71, can produce an optimal policy in a model-free way based only on observed trajectories.

In this paper, we synthesize the work on risk-aware optimization with reinforcement learning, specifically, $Q$-learning. As our main contribution, we develop a novel asynchronous stochastic-approximation type algorithm to solve infinite-horizon risk-aware MDPs. This algorithm can compute the risk-aware optimal policy based only on observations, without any knowledge of the explicit form of the cost function or the transition probabilities.

\section{$1.1 \quad$ Literature review}

\subsubsection{Risk measures}

In general, a risk measure is a mapping from random variables to scalars. It can be interpreted as the amount of an asset (traditionally currency) to be kept in reserve to make the risk acceptable. The literature emphasizes convex and coherent risk measures. In [58], a theory of convex analysis is developed for optimization

\footnotetext{
*Wenjie Huang (wenjiehuang@cuhk.edu.cn) is an International Postdoctoral Fellow at Shenzhen Research Institute of Big Data (SRIBD) and Institute for Data and Decison Analytics, The Chinese University of Hong Kong, Shenzhen. His research was supported by SRIBD International Postdoctoral Fellowship, and the National Research Foundation (NRF), Prime Ministers Office, Singapore under its Campus for Research Excellence and Technological Enterprise (CREATE) program.

${ }^{\dagger}$ William B. Haskell (whaskell@purdue.edu) is an Assistant Professor in Krannert School of Management at Purdue University. His research was supported by Singapore Ministry of Education Grant R-266-000-083-133 and by Singapore Ministry of Education Tier II Grant MOE2015-T2-2-148.
} 
of convex risk measures. Several specific examples of convex and coherent risk measures along with their various risk envelopes are given in [63, Chapter 6].

Special attention has been given to the class of coherent and law invariant risk measures, the most well known being conditional value-at-risk (CVaR). Optimization of CVaR is studied in [56], which reveals that CVaR has many desirable properties for stochastic optimization. The most famous representation result for law-invariant coherent risk measures is the Kusuoka representation (see [61] for example) which shows that such risk measures can be 'built' out of CVaR. There are several other important classes of risk measures such as: optimized certainty equivalent [13], spectral risk measures [2], distortion risk measures [15], and entropic risk measures 27 .

Numerical methods for risk-aware optimization are critical for practical application. In [38], CVaRconstrained optimization problems are solved with a combination of discretization, linearization, and sample average approximation. For multistage CVaR optimization, 50] uses the dual representation of general coherent risk measures to develop sampling-based algorithms. In [7], stochastic approximation is used to estimate $\mathrm{CVaR}$ in data-driven optimization. In addition, in [23, stochastic interior-point algorithms are developed for risk-constrained optimization.

\subsubsection{Risk-aware MDPs}

Risk-aware MDPs have been widely studied. In [8], the authors minimize the conditional value-at-risk of the discounted cost over both the finite and infinite horizon. In the follow-up work [9], the authors minimize a certainty equivalent of the total cost for both finite and infinite horizon problems. Dynamic programming methods are developed in both [8] and [9]. A CVaR-constrained MDP is solved with both offline and online iterative algorithms in [16. In [24], both risk and modeling errors are taken into account in an MDP framework for risk-sensitive and robust decision-making, and an approximate value-iteration type algorithm is presented.

In [30], the authors study stochastic dominance-constrained MDPs, and show that this class of MDPs can be reformulated as linear programming problems using the convex analytic approach. In [31], the authors develop the convex analytic approach for a general family of risk-aware MDPs.

Dynamic programming equations are developed for a wide class of risk-aware MDPs in [57, and corresponding value iteration and policy iteration algorithms are developed. The family of risk measures studied in this work are often called "dynamic risk measures" or "Markov risk measures", and are notable for satisfying the property of time-consistency. In [65], the theory of risk-sensitive MDPs is developed based on iterative risk measures which only depend on the current state, rather than on the whole history.

In [24, reinforcement learning algorithms for percentile risk-constrained MDPs are proposed. In [53 67, policy gradient algorithms are applied to MDPs with CVaR appearing in either the objective or constraints. In [36], a specific class of risk measures called quantile-based risk measures is proposed for MDPs and a simulation-based approximate dynamic programming (ADP) algorithm is developed for the resulting problem. This paper emphasizes importance sampling, to direct samples toward the risky region as the ADP algorithm progresses. In [66, a risk-sensitive reinforcement learning algorithm based on utility functions is investigated. A similar technique is applied to the risk-sensitive control of finite MDPs in 17.

\subsubsection{Stochastic approximation and $Q$-learning}

$Q$-learning is introduced in [71]. The idea of $Q$-learning is to use the observed transitions and costs to compute the optimal policy (so that exact knowledge of the underlying MDP model is not needed). In [69], a thorough convergence proof of the $Q$-learning algorithm is given based on stochastic approximation and the theory of parallel asynchronous algorithms (see [18] for more details on the theory of stochastic approximation). $Q$ learning has wide applications in the areas of robotics and operations management, and has also recently been applied to stochastic games 33]. Stochastic approximation has also been applied to solve static stochastic optimization problems. In 44,45, efficient and robust stochastic approximation algorithms are developed to solve saddle-point problem and optimize non-smooth functions. 


\subsection{Contributions}

As our main contribution, we develop a stochastic approximation-type algorithm for infinite-horizon riskaware MDPs that covers a wide range of risk measures. This algorithm is model-free and it can compute the risk-aware optimal policy based only on observations. We make the following three specific contributions:

1. Generality of risk measures: There exists literature (e.g. [17, 66]) studying reinforcement learning for risk-sensitive MDPs. The "risk-sensitive" objective in [17] specifically refers to the expectation of the exponential function of cumulative costs. In [66], the "risk-sensitive" objective is essentially utilitybased shortfall, and in [9], "risk-sensitive" refers to utility-based certainty equivalent. To the best of our knowledge, our present paper adds to the literature by incorporating saddle-point risk measures. In [36], only quantile-based risk measures are included. In [8, 67, the algorithms are specific to CVaR.

2. Model-free asynchronous algorithm: There exist several dynamic programming based algorithms for solving risk-aware MDPs (see $8,36,57,65,67]$ ), but they all rely on some information about the underlying transitions or cost function. Our novel stochastic approximation algorithm is completely model-free. Our algorithm is also asynchronous, which means that the $Q$-value is only updated when the corresponding state-action pair is explored. This algorithm works even when no prior information on the underlying MDP is available.

3. Explicit sample complexity results: We give a detailed convergence rate analysis of our algorithm for both polynomial and linear learning rates. We also show numerically that the convergence rate of our algorithm is close to that of standard $Q$-learning. In [17,36, the almost sure convergence of the proposed algorithms is demonstrated, but the explicit convergence rates are not derived.

This paper is organized as follows. Section 2 reviews preliminaries on risk measures and risk-aware MDPs. Section 3 then introduces saddle-point risk measures and shows by example that many widely investigated risk measures fall within this framework. Section 4 presents the details of our algorithm as well as its almost sure convergence and convergence rate. Section 5 contains the proofs of all our main theorems. We report numerical experiments in Section 6 and then conclude the paper in Section 7.

\section{Preliminaries}

This section introduces preliminary concepts and notations (listed in Table 1).

\section{$2.1 \quad$ Risk measures}

We begin with a probability space $(\Omega, \mathcal{F}, P)$, where $\Omega$ is a sample space, $\mathcal{F}$ is a $\sigma$-algebra on $\Omega$, and $P$ is a probability measure on $(\Omega, \mathcal{F})$. We work in $\mathcal{L}=L_{\infty}(\Omega, \mathcal{F}, P)$, the space of essentially bounded $\mathcal{F}$-measurable mappings. For $X, Y \in \mathcal{L}, Y \succeq X$ means that $Y(\omega) \geq X(\omega)$ for all $\omega \in \Omega$.

We define a risk measure to be a function $\rho: \mathcal{L} \rightarrow \mathbb{R}$, which assigns to a random variable $X \in \mathcal{L}$ a real scalar value $\rho(X)$. The following are four key properties of risk measures:

(A1) Monotonicity: If $X \succeq Y$, then $\rho(X) \geq \rho(Y)$.

(A2) Translation Invariance: $\rho(X+r)=\rho(X)+r$ for $r \in \mathbb{R}$.

(A3) Convexity: $\rho(\lambda X+(1-\lambda) Y) \leq \lambda \rho(X)+(1-\lambda) \rho(Y)$ for $0 \leq \lambda \leq 1$.

(A4) Positive Homogeneity: $\rho(\alpha X)=\alpha \rho(X)$ for $\alpha \geq 0$.

These conditions were introduced in the pioneering paper [4] and have since been heavily justified in other work including 15 42 58. Property (A1) states that a random variable with greater cost almost surely must have higher risk. (A2) states that the addition of a certain cost increases the risk by the same amount. (A3) gives precise meaning to the idea that diversification should not increase risk. (A4) states that the risk of a position is proportional to its size (i.e., if we double our cost then we double our risk). A risk measure satisfying properties (A1)-(A3) is called a convex risk measure, and a risk measure satisfying properties (A1)-(A4) is called a coherent risk measure. 
Table 1: List of Key Notation

\begin{tabular}{cc}
\hline Notations & Definitions \\
\hline$N, n$ & Outer iterations \\
$T, t$ & Inner iterations \\
$G$ & Objective function for saddle-point risk measure \\
$\mathbb{S}, \mathbb{A}$ & State and action space \\
$\mathcal{T}, \mathcal{T}_{G}$ & Risk-aware Bellman operator \\
$\mathcal{R}_{(s, a)}^{G}$ & Risk measure with respect to state-action pair $(s, a)$ and function $G$ \\
$\mathcal{Y}, \mathcal{Z}$ & Compact sets \\
$K_{\mathcal{Y}}, K_{\mathcal{Z}}$ & Euclidean diameters of $\mathcal{Y}$ and $\mathcal{Z}$. \\
$L$ & Bounds for the subgradients \\
$K_{G}$ & Constant of Lipschitz continuity for function $G$ \\
$K_{S}$ & Stability modulus of saddle-point \\
$\mathcal{G}_{t}^{n}$ & The history of RaQL for $t \leq T$ and $n \leq N$ \\
$\epsilon_{t}^{n}$ & Risk estimation error, for $t \leq T$ and $n \leq N$ \\
$\xi_{t}^{n}$ & Approximation error, for $t \leq T$ and $n \leq N$ \\
$\tau$ & The iteration w.r.t sequence $D$ \\
$\tau_{m}$ & The iteration when the approximation error of $Q$-value is bounded by $D_{m}$ \\
$D_{m}$ & A constructed sequence $D$ with time horizon $m$ \\
$Z_{t}^{n+1, \tau}, Y_{t}^{n+1, \tau}$ & Two random processes decomposed from $\left\{Q_{t}^{n}\right\}$ \\
$\beta_{T}$ & Discount factor of sequence $D_{m}$ \\
$e$ & Natural logarithm \\
$\|\cdot\|_{2}$ & Infinite norm \\
$\|\cdot\|_{\infty}$ & $L_{2}$-norm \\
\hline
\end{tabular}

\subsection{Risk-aware MDPs}

A MDP is given by the tuple $(\mathbb{S}, \mathbb{A}, P, c)$ where $\mathbb{S}$ and $\mathbb{A}$ are the state and action spaces and $\mathbb{K}:=$ $\{(s, a) \in \mathbb{S} \times \mathbb{A}\}$ is the set of all state-action pairs. Let $\mathcal{P}(\mathbb{S})$ be the space of probability measures over $\mathbb{S}$, and define $\mathcal{P}(\mathbb{A})$ similarly. The transition law $P$ governs the system evolution where $P(\cdot \mid s, a) \in \mathcal{P}(\mathbb{S})$ for all $(s, a) \in \mathbb{K}$, i.e., $P\left(s^{\prime} \mid s, a\right)$ for $s^{\prime} \in \mathbb{S}$ is the probability of next visiting state $s^{\prime}$ given the current stateaction pair $(s, a)$. The cost function $c: \mathbb{K} \rightarrow \mathbb{R}$ gives the cost of each state-action pair. Finally, $\gamma \in(0,1)$ is the discount factor. Let $\phi: \mathbb{S} \rightarrow \mathcal{P}(\mathbb{A})$ be a randomized policy. For a given $\phi$, we obtain a stochastic process $\left\{\left(s_{t}, a_{t}\right)\right\}_{t \geq 0}$ where $s_{t}$ and $a_{t}$ are the state and action at stage $t$, respectively.

We make the following assumptions.

Assumption 2.1. (i) $\mathbb{S}$ and $\mathbb{A}$ are finite.

(ii) $0 \leq c(s, a) \leq C_{\max }$ for all $(s, a) \in \mathbb{K}$. Set $V_{\max }:=C_{\max } /(1-\gamma)$.

Many real life MDPs satisfy Assumption 2.1(i), including machine replacement and sequential online auctions [34], critical infrastructure protection [48], wireless sensor networks [3], and human-robot interaction systems [37, 39].

In [57], the modern theory of risk measures is adapted to MDPs. This class of risk-aware MDPs is constructed in the following way. Denote our sequence of costs as $X_{t}=c\left(s_{t}, a_{t}\right)$ for all $t \geq 0$. We begin by formalizing some details about the risk of finite cost sequences $X_{t, T}:=\left(X_{t}, X_{t+1}, \ldots, X_{T}\right)$ before we consider the risk of the infinite cost sequence $X_{0}, X_{1}, \ldots$ actually faced by the controller. Let $\mathcal{L}_{t}:=\mathcal{L}_{\infty}\left(\Omega, \mathcal{F}_{t}, P\right)$ and $\mathcal{L}_{t, T}:=\mathcal{L}_{t} \times \mathcal{L}_{t+1} \times \cdots \times \mathcal{L}_{T}$ for all $0 \leq t \leq T<\infty$.

Definition 2.2. 57. Definition 1] For fixed $T \geq 1$ and $0 \leq t \leq T$, (i) A mapping $\rho_{t, T}: L_{t, T} \rightarrow L_{t}$, is called a conditional risk measure if: $\rho_{t, T}\left(Z_{t, T}\right) \leq \rho_{t, T}\left(X_{t, T}\right)$ for all $Z_{t, T}, X_{t, T} \in \mathcal{L}_{t, T}$ such that $Z_{t, T} \leq X_{t, T}$.

(ii) A dynamic risk measure is a sequence of conditional risk measures $\left\{\rho_{t, T}\right\}_{t=0}^{T}$.

We now make our key assumptions about dynamic risk measures. 
Assumption 2.3. For fixed $T \geq 1$ and $0 \leq t \leq T$, suppose the dynamic risk measure $\left\{\rho_{t, T}\right\}_{t=0}^{T}$ satisfies the following conditions:

(i) (Normalization) $\rho_{t, T}(0,0, \ldots, 0)=0$.

(ii) (Conditional translation invariance) For any $X_{t, T} \in \mathcal{L}_{t, T}$,

$$
\rho_{t, T}\left(X_{t}, X_{t+1}, \ldots, X_{T}\right)=X_{t}+\rho_{t, T}\left(0, X_{t+1}, \ldots, X_{T}\right) .
$$

(iii) (Convexity) For any $X_{t, T}, Y_{t, T} \in \mathcal{L}_{t, T}$ and $0 \leq \lambda \leq 1, \rho_{t, T}\left(\lambda X_{t, T}+(1-\lambda) Y_{t, T}\right) \leq \lambda \rho_{t, T}\left(X_{t, T}\right)+$ $(1-\lambda) \rho_{t, T}\left(Y_{t, T}\right)$.

(iv) (Positive homogeneity) For any $X_{t, T} \in \mathcal{L}_{t, T}$ and $\alpha \geq 0, \rho_{t, T}\left(\alpha X_{t, T}\right)=\alpha \rho_{t, T}\left(X_{t, T}\right)$.

(v) (Time-consistency) For any $X_{t, T}, Y_{t, T} \in \mathcal{L}_{t, T}$ and $0 \leq \tau \leq \theta \leq T$, the conditions $X_{k}=Y_{k}$ for $k=\tau, \ldots, \theta-1$ and $\rho_{\theta, T}\left(X_{\theta}, \ldots, X_{T}\right) \leq \rho_{\theta, T}\left(Y_{\theta}, \ldots, Y_{T}\right)$ imply $\rho_{\tau, T}\left(X_{\tau}, \ldots, X_{T}\right) \leq \rho_{\tau, T}\left(Y_{\tau}, \ldots, Y_{T}\right)$.

Many of these properties (monotonicity, convexity, positive homogeneity, and translation invariance) were originally introduced for static risk measures as properties (A1)-(A4). The next theorem gives a recursive formulation for dynamic risk measures satisfying Assumption 2.3 This representation is the foundation of [57] and subsequent work on time-consistent dynamic risk measures. To express this result, we define a mapping $\rho_{t}: \mathcal{L}_{t+1} \rightarrow \mathcal{L}_{t}$ for $t \geq 0$ to be a one-step (conditional) risk measure if $\rho_{t}\left(X_{t+1}\right)=\rho_{t, t+1}\left(0, X_{t+1}\right)$.

Theorem 2.4. [57, Theorem 1] Suppose Assumption 2.3 holds, then

$$
\rho_{t, T}\left(X_{t}, X_{t+1}, \ldots, X_{T}\right)=X_{t}+\rho_{t}\left(X_{t+1}+\rho_{t+1}\left(X_{t+2}+\cdots+\rho_{T}\left(X_{T}\right)\right)\right),
$$

for all $0 \leq t \leq T$, where $\rho_{t}, \ldots, \rho_{T}$ are one-step risk measures.

Now we consider the risk of an infinite cost sequence. Following [57], the discounted measure of risk $\rho_{t, T}^{\gamma}: \mathcal{L}_{t, T} \rightarrow \mathbb{R}$ is defined via

$$
\rho_{t, T}^{\gamma}\left(X_{t}, X_{t+1}, \ldots, X_{T}\right):=\rho_{t, T}\left(\gamma^{t} X_{t}, \gamma^{t+1} X_{t+1}, \ldots, \gamma^{T} X_{T}\right) .
$$

Define $\mathcal{L}_{t, \infty}:=\mathcal{L}_{t} \times \mathcal{L}_{t+1} \times \cdots$ for $t \geq 0$ and $\rho^{\gamma}: \mathcal{L}_{0, \infty} \rightarrow \mathbb{R}$ via

$$
\rho^{\gamma}\left(X_{0}, X_{1}, \ldots\right):=\lim _{T \rightarrow \infty} \rho_{0, T}^{\gamma}\left(X_{0}, X_{1}, \ldots\right) .
$$

To provide our final representation result, we introduce the additional assumption that risk preferences are stationary (they only depend on the sequence of costs ahead, and are independent of the current time).

Assumption 2.5. (Stationary preferences) For all $T \geq 1$ and $s \geq 0$,

$$
\rho_{0, T}^{\gamma}\left(X_{0}, X_{1}, \ldots, X_{T}\right)=\rho_{s, T+s}^{\gamma}\left(X_{0}, X_{1}, \ldots, X_{T}\right) .
$$

When Assumptions 2.3 and 2.5 are satisfied, the corresponding dynamic risk measure is given by the recursion:

$$
\rho^{\gamma}\left(X_{0}, X_{1}, \ldots, X_{T}, \ldots\right)=X_{0}+\rho_{1}\left(\gamma X_{1}+\rho_{2}\left(\gamma^{2} X_{2}+\cdots+\rho_{T}\left(\gamma^{T} X_{T}\right)+\cdots\right)\right),
$$

where $\rho_{1}, \rho_{2}, \ldots$ are all one-step risk measures. Based on representation 2.2 , we may evaluate the risk of a policy $\phi$ via

$$
J\left(\phi, s_{0}\right):=\rho\left(c\left(s_{0}, a_{0}\right)+\gamma \cdot \rho\left(c\left(s_{1}, a_{1}\right)+\gamma \cdot \rho\left(c\left(s_{2}, a_{2}\right)+\cdots\right)\right)\right),
$$

where $s_{0}$ is the initial state. To clarify, the same one-step risk measure $\rho$ appears at all times $t \geq 0$ due to the property of stationarity. Formulation (2.3) explicitly captures the risk with respect to the cost associated with the current state-action pair, as well as the future risk. Let $\Pi$ denote the class of deterministic stationary policies $\pi$ which map from states to actions, i.e., $\pi: \mathbb{S} \rightarrow \mathbb{A}$. From [57, Theorem 4], it shows that there exists an optimal deterministic stationary policy that minimizes Eq. (2.3). The corresponding risk-aware MDP is

$$
\min _{\pi \in \Pi} J\left(\pi, s_{0}\right)
$$




\section{Saddle-point risk measures}

This section introduces the saddle-point representation of risk measures. We elaborate on two main reasons for choosing this representation. First, many widely investigated risk measures can be represented as stochastic saddle-point problems including: conditional value-at-risk, optimized certainty equivalent, absolute semi-deviation, and functionally coherent risk measures. Second, there are efficient algorithms for solving stochastic saddle-point problems (see [44,45]) and thus for computing the risk.

To proceed, we now assume that $\Omega$ is Borel measurable and $\mathcal{L}$ is the set of all $X$ with bounded support $\left[\eta_{\min }, \eta_{\max }\right]$ and $\eta_{\min }, \eta_{\max }$ satisfying $-\infty<\eta_{\min }<\eta_{\max }<\infty$ (i.e., $X(\omega) \in\left[\eta_{\min }, \eta_{\max }\right]$ for all $\omega \in \Omega$ ). Take $\mathcal{Y} \subset \mathbb{R}^{d_{1}}$ and $\mathcal{Z} \subset \mathbb{R}^{d_{2}}$ to be closed and convex sets and define $K_{\mathcal{Y}}, K_{\mathcal{Z}}$ to be the Euclidean diameters of $\mathcal{Y}$ and $\mathcal{Z}$, respectively. For a proper function $G: \mathcal{L} \times \mathcal{Y} \times \mathcal{Z} \rightarrow \mathbb{R}$, we consider the risk measure:

$$
\rho(X)=\max _{z \in \mathcal{Z}} \min _{y \in \mathcal{Y}} \mathbb{E}_{P}[G(X, y, z)]
$$

We define $\partial_{y} G(\cdot, y, z)$ and $\partial_{z} G(\cdot, y, z)$ to be the subdifferentials of $G$ for all $(y, z) \in \mathcal{Y} \times \mathcal{Z}$, and we define $G_{y}(\cdot, y, z) \in \partial_{y} G(\cdot, y, z)$ and $G_{z}(\cdot, y, z) \in \partial_{z} G(\cdot, y, z)$ to be particular subgradients with respect to $y$ and $z$. We make the following assumptions on the function $G$.

Assumption 3.1. [45, Assumption $B]$ (i) $\omega \rightarrow G(X(\omega), y, z)$ is P-square summable for every $y \in \mathcal{Y}$ and $z \in \mathcal{Z}$, i.e., $\int_{\Omega}|G(X(\omega), y, z)|^{2} P(d \omega)<\infty$, for all $(y, z) \in \mathcal{Y} \times \mathcal{Z}$.

(ii) $G$ is Lipschitz continuous on $\mathcal{L} \times \mathcal{Y} \times \mathcal{Z}$ with constant $K_{G}>1$.

(iii) $y \rightarrow G(X, y, z)$ is convex and $z \rightarrow G(X, y, z)$ is concave for all $(X, y, z) \in \mathcal{L} \times \mathcal{Y} \times \mathcal{Z}$.

(iv) Any selection of subgradients $\omega \rightarrow G_{y}(X(\omega), y, z)$ and $\omega \rightarrow G_{z}(X(\omega), y, z)$ is Borel measurable. The subgradients $G_{y}(X, y, z)$ and $G_{z}(X, y, z)$ are uniformly bounded, i.e., there exists $L>0$ such that $\left\|G_{y}(X, y, z)\right\|_{2} \leq L$ and $\left\|G_{z}(X, y, z)\right\|_{2} \leq L$ for all $(X, y, z) \in \mathcal{L} \times \mathcal{Y} \times \mathcal{Z}$.

Under the assumption that $G$ is proper on $\mathcal{L} \times \mathcal{Y} \times \mathcal{Z}$ and Assumption 3.1.(iii), we know that the subdifferentials $\partial_{y} G(\cdot, y, z)$ and $\partial_{z} G(\cdot, y, z)$ are non-empty for all $(y, z) \in \mathcal{Y} \times \mathcal{Z}$ by [55. Theorem 23.4]. Based on 63 . Theorem 7.47] and [63, Remark 18], under Assumption 3.1 (i), the subdifferentials $\partial_{y} \mathbb{E}_{P}[G(X, y, z)]$ and $\partial_{z} \mathbb{E}_{P}[G(X, y, z)]$ are nonempty and satisfy $\partial_{y} \mathbb{E}_{P}[G(X, y, z)]=\mathbb{E}_{P}\left[\partial_{y} G(\cdot, y, z)\right]$, and $\partial_{z} \mathbb{E}_{P}[G(X, y, z)]=$ $\mathbb{E}_{P}\left[\partial_{z} G(\cdot, y, z)\right]$, for all $x$ and $y$ in the interior of $\mathcal{Y}$ and $\mathcal{Z}$, respectively. Thus, the subgradients $G_{y}(X, y, z)$ and $G_{z}(X, y, z)$ satisfy $\mathbb{E}_{P} G_{y}(X, y, z) \in \partial_{y} \mathbb{E}_{P}[G(X, y, z)]$ and $\mathbb{E}_{P} G_{z}(X, y, z) \in \partial_{z} \mathbb{E}_{P}[G(X, y, z)]$. Under Assumptions 3.1(i) and (iv), we see that $\left\|\mathbb{E}_{P} G_{y}(X, y, z)\right\|_{2}$ and $\left\|\mathbb{E}_{P} G_{z}(X, y, z)\right\|_{2}$ are both bounded by $L$.

The following Theorem 3.2 provides sufficient conditions for the saddle-point risk measure (3.1) to be a convex risk measure satisfying axioms (A1)-(A3). In particular, we can find a special class of functions

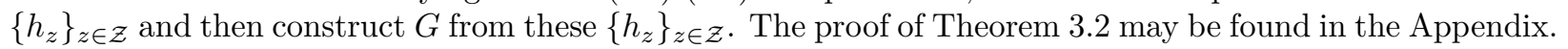

Theorem 3.2. Set $\mathcal{Y}=\left[\eta_{\min }, \eta_{\max }\right]$ and let $\left\{h_{z}\right\}_{z \in \mathcal{Z}}$ be a collection of functions such that $h_{z}(X, y)$ (for $X \in \mathcal{L}$ and $y \in \mathcal{Y})$, parameterized by $z \in \mathcal{Z}$ that satisfies:

(i) $\omega \rightarrow h_{z}(X(\omega), y)$ is $P$-square summable for every $y \in \mathcal{Y}$ and $z \in \mathcal{Z}$.

(ii) $h_{z}(X, y)$ is convex in $y \in \mathcal{Y}$ and concave in $z \in \mathcal{Z}$, for all $X \in \mathcal{L}$.

(iii) Any selection of subgradients of $h_{z}(X, y)$ with respect to $z \in \mathcal{Z}$ and $y \in \mathcal{Y}$ is Borel measurable and uniformly bounded for all $X \in \mathcal{L}$.

(iv) $h_{z}$ is Lipschitz continuous on $\mathcal{L} \times \mathcal{Y}$ with constant $K_{G}-1$ for all $z \in \mathcal{Z}$. Then,

$$
G(X, y, z)=y+h_{z}(X, y), y \in \mathcal{Y}, z \in \mathcal{Z}
$$

satisfies Assumption 3.1. Further, formulation (3.1) with the choice of (3.2) is a convex risk measure satisfying axioms (A1)-(A3).

We now detail several applications of Theorem 3.2

Example 3.3. Optimized certainty equivalent (OCE, see $[13])$. Define $\mathcal{Y}=\left[\eta_{\min }, \eta_{\max }\right](\mathcal{Z}$ is a singleton). First, we construct CVaR by choosing:

$$
h_{z}(X, y)=-\frac{1}{(1-\alpha)} \max \{X-y, 0\}, \alpha \in[0,1], \forall z \in \mathcal{Z} .
$$


We then obtain:

$$
G(X, y, z)=y+(1-\alpha)^{-1} \mathbb{E}_{P}[\max \{X-y, 0\}], \forall z \in \mathcal{Z},
$$

and

$$
\operatorname{CVaR}_{\alpha}(X):=\min _{\eta \in\left[\eta_{\min }, \eta_{\max }\right]} \mathbb{E}[G(X, y, z)]=\min _{\eta \in\left[\eta_{\min }, \eta_{\max }\right]} \mathbb{E}\left[y+h_{z}(X, y)\right], \forall z \in \mathcal{Z} .
$$

We can generalize CVaR to OCE by substituting a general utility function in place of (3.3). Define $u$ : $\mathbb{R} \rightarrow[0,1]$ to be a concave utility function that is differentiable with bounded derivative $\nabla u(\cdot)$. In this case, $h_{z}(X, y)=u(y-X)$, for all $z \in \mathcal{Z}$, and the function $G$ is:

$$
G(X, y, z)=y-\mathbb{E}_{P}[u(y-X)], \forall z \in \mathcal{Z} .
$$

Example 3.4. Absolute semi-deviation is a type of mean-risk model. The absolute semi-deviation is $\rho_{A S}(X):=\mathbb{E}[X]+\iota \mathbb{E}\left[\left(X-\mathbb{E}([X])_{+}\right]\right.$for the weight coefficient $\iota \in[0,1]$ (see $[63)$. Define $\mathcal{Y}=\left[\eta_{\min }, \eta_{\max }\right]$, and $\mathcal{Z}=[0,1]$. By 63 . Chapter 6.5.2], we have: $h_{z}(X, y)=(1-\iota z) X+\iota(X-y)_{+}+\iota z-1$,

$$
G(X, y, z)=X+\iota(X-y)_{+}+\iota z(y-X),
$$

and

$$
\rho_{\mathrm{AS}}(X):=\min _{y \in\left[\eta_{\min }, \eta_{\max }\right]} \max _{z \in[0,1]} \mathbb{E}[G(X, y, z)]=\min _{y \in\left[\eta_{\min }, \eta_{\max }\right]} \max _{z \in[0,1]} \mathbb{E}\left[y+h_{z}(X, y)\right] .
$$

Example 3.5. The functionally coherent risk measure (see 46 47]) is a finite version of the Kusuoka representation (see e.g. [61]), which is the weighted average multiple CVaR in terms of their confidence levels. Given a range of confidence levels [0,1) with $\left\{\alpha_{i}\right\}_{i=0}^{m} \subset[0,1)$ and $0 \leq \alpha_{0}<\alpha_{1}<\cdots<\alpha_{m}<1$, we define $\mathcal{P}\left(\left\{\alpha_{i}\right\}_{i=1}^{m}\right)$ to be the set of probability distributions on $\left\{\alpha_{i}\right\}_{i=1}^{m}$, and we let $\mathfrak{M}$ be a closed convex subset of $\mathcal{P}\left(\left\{\alpha_{i}\right\}_{i=1}^{m}\right)$. In this case, we let $z=\left(z_{1}, \ldots, z_{m}\right) \in \mathbb{R}^{m}, y=\left(y_{1}, \ldots, y_{m}\right) \in \mathbb{R}^{m}$, and $h_{z}(X, y)=$ $\sum_{i=1}^{m} z_{i}\left(1-\alpha_{i}\right)^{-1} \max \{X-y, 0\}, \mathcal{Y}=\left[\eta_{\min }, \eta_{\max }\right]^{m}$, and $\mathcal{Z}=\mathfrak{M}$, and

$$
G(X, y, z)=\sum_{i=1}^{m} z_{i}\left\{y_{i}+\left(1-\alpha_{i}\right)^{-1} \mathbb{E}_{P}\left[\max \left\{X-y_{i}, 0\right\}\right]\right\}
$$

We then obtain

$$
\rho_{\mathrm{KS}}(X):=\min _{y \in\left[\eta_{\min }, \eta_{\max }\right]^{m}} \max _{z \in \mathfrak{M}} \mathbb{E}[G(X, y, z)]=\min _{y \in\left[\eta_{\min }, \eta_{\max }\right]^{m}} \max _{z \in \mathfrak{M}} \mathbb{E}\left[y+h_{z}(X, y)\right] .
$$

Each instance of $G$ constructed in Examples 3.3, 3.5 and 3.4, satisfies parts (i)-(iv) in Assumption 3.1

\section{Risk-aware Q-learning algorithm}

In this section, we introduce our 'Risk-aware Q-learning' (RaQL) algorithm. RaQL is an asynchronous offpolicy learning algorithm with an inner and outer loop structure. It uses stochastic approximation in the inner loop for risk estimation and $Q$-learning in the outer loop for computing the optimal risk-aware policy. The "off-policy" characteristic means that the policy for exploring new states (denoted $\bar{\pi}$ ) and the policy $\pi$ from the $Q$-value updates are different. The "asynchronous" characteristic means that the step-size rule of the algorithm ensures that only a single state-action pair is updated when it is observed and sends the step-size to zero whenever a state-action pair is not visited.

\subsection{Algorithm description}

Let $\mathcal{V} \subset \mathbb{R}^{|\mathbb{S}|}$ be the space of value functions on $\mathbb{S}$ equipped with the supremum norm $\|v\|_{\infty}:=\max _{s \in \mathbb{S}}|v(s)|$. Under Assumption 2.1. we have $\|v\|_{\infty} \leq V_{\max }=C_{\max } /(1-\gamma)$ for all $v \in \mathcal{V}$. The risk-aware Bellman operator $\mathcal{T}: \mathcal{V} \rightarrow \mathcal{V}$ corresponding to the MDP (2.4) is

$$
[\mathcal{T} v](s):=\min _{a \in \mathbb{A}}\left\{c(s, a)+\gamma \rho\left(v\left(s^{\prime}\right)\right)\right\}, \forall s \in \mathbb{S},
$$


where $s^{\prime}$ is the random next state following the transition kernel $P(\cdot \mid s, a)$. By [57, Theorem 4] and 65 , Theorem 5.5], $\mathcal{T}$ is a contraction with respect to the supremum norm and Problem (2.4) has an optimal value function $v^{*}$ satisfying $v^{*}=\mathcal{T} v^{*}$. The following Proposition 4.1 demonstrates why $\mathcal{T}$ is a contraction when $\rho$ is a convex risk measure.

Proposition 4.1. Suppose $\rho$ is a convex risk measure, then

$$
\left\|\mathcal{T} v_{1}-\mathcal{T} v_{2}\right\|_{\infty} \leq \gamma\left\|v_{1}-v_{2}\right\|_{\infty}
$$

for all $v_{1}, v_{2} \in \mathcal{V}$.

Proof. By [22, 28, 58], any convex risk measure $\rho$ can be represented as

$$
\rho(X)=\sup _{P \in \mathcal{P}(\Omega)}\left\{\mathbb{E}_{P}[X]-\mu(P)\right\}
$$

where $\mu$ is a convex function satisfying $\inf _{P \in \mathcal{P}(\Omega)} \mu(P)=0$, and $P(\Omega)$ is the set of probability distributions on $(\Omega, \mathcal{F})$. Then, since $\rho$ is convex risk measure,

$$
\begin{aligned}
\left\|\mathcal{T} v_{1}-\mathcal{T} v_{2}\right\|_{\infty} & \leq \gamma\left|\sup _{P \in \mathcal{P}(\Omega)}\left\{\mathbb{E}_{P}\left[v_{1}\right]-\mu(P)\right\}-\sup _{P \in \mathcal{P}(\Omega)}\left\{\mathbb{E}_{P}\left[v_{2}\right]-\mu(P)\right\}\right| \\
& \leq \gamma\left|\sup _{P \in \mathcal{P}(\Omega)} \mathbb{E}_{P}\left[v_{1}-v_{2}\right]\right| \leq \gamma \sup _{P \in \mathcal{P}(\Omega)} \mathbb{E}_{P}\left|v_{1}-v_{2}\right| \leq \gamma\left\|v_{1}-v_{2}\right\|_{\infty},
\end{aligned}
$$

since $\mathbb{E}_{P^{\prime}}\left|v_{1}-v_{2}\right| \leq\left\|v_{1}-v_{2}\right\|_{\infty}$ for any $P \in \mathcal{P}(\Omega)$.

For the dynamic setting, we now introduce the risk measure 3.1 for each state-action pair. Given the current state $s \in \mathbb{S}$ and action $a \in \mathbb{A}$, the risk for the value of the next state $s^{\prime} \in \mathbb{S}$ is defined to be:

$$
\mathcal{R}_{(s, a)}^{G}\left(v\left(s^{\prime}\right)\right):=\min _{y \in \mathcal{Y}} \max _{z \in \mathcal{Z}} \mathbb{E}_{s^{\prime} \sim P(\cdot \mid s, a)}\left[G\left(v\left(s^{\prime}\right), y, z\right)\right],
$$

where the expectation is with respect to the transition kernel $P(\cdot \mid s, a)$. Throughout the remainder of this paper, we assume that $\mathcal{R}_{(s, a)}^{G}$ is a convex risk measure satisfying axioms (A1)-(A3) for all $(s, a) \in \mathbb{K}$, which means that $G$ may be constructed from Theorem 3.2 For simpler notation, we just take $G$ in 4.3 to be the same for all state-action pairs $(s, a) \in \mathbb{K}$. We also assume that the $G$ in 4.3 satisfies Assumption 3.1 The corresponding risk-aware Bellman operator is then $\mathcal{T}_{G}: \mathcal{V} \rightarrow \mathcal{V}$ defined by

$$
\left[\mathcal{T}_{G} v\right](s):=\min _{a \in \mathbb{A}}\left\{c(s, a)+\gamma \mathcal{R}_{(s, a)}^{G}\left(v\left(s^{\prime}\right)\right)\right\}, \forall s \in \mathbb{S} .
$$

Since $\mathcal{T}_{G}$ is a contraction operator, Problem 2.4 has an optimal value function $v^{*}$ satisfying $v^{*}=\mathcal{T}_{G} v^{*}$. Additionally, based on [57, Theorem 4] and [65. Theorem 5.5], Problem (2.4 has a stationary optimal policy $\pi^{*} \in \Pi$ which is greedy with respect to $v^{*}$, i.e.

$$
\pi^{*}(s) \in \arg \min _{a \in \mathbb{A}}\left\{c(s, a)+\gamma \mathcal{R}_{(s, a)}^{G}\left(v^{*}\left(s^{\prime}\right)\right)\right\}, \forall s \in \mathbb{S} .
$$

Now, based on [71] and 66. Theorem 1], we define the risk-aware $Q$-value to be:

$$
Q(s, a):=c(s, a)+\gamma \mathcal{R}_{(s, a)}^{G}\left(\min _{a^{\prime} \in \mathbb{A}} Q\left(s^{\prime}, a^{\prime}\right)\right), \forall(s, a) \in \mathbb{K},
$$

and the optimal risk-aware $Q$-value, denoted as $Q^{*}$, to be:

$$
Q^{*}(s, a):=c(s, a)+\gamma \mathcal{R}_{(s, a)}^{G}\left(\max _{a^{\prime} \in \mathbb{A}} Q^{*}\left(s^{\prime}, a^{\prime}\right)\right), \forall(s, a) \in \mathbb{K} .
$$

The procedure of RaQL is presented as Algorithm 1 (we provide the pseudo code in Algorithm 1 and later give the detailed descriptions of each step). RaQL is an asynchronous algorithm based on two loops: an 


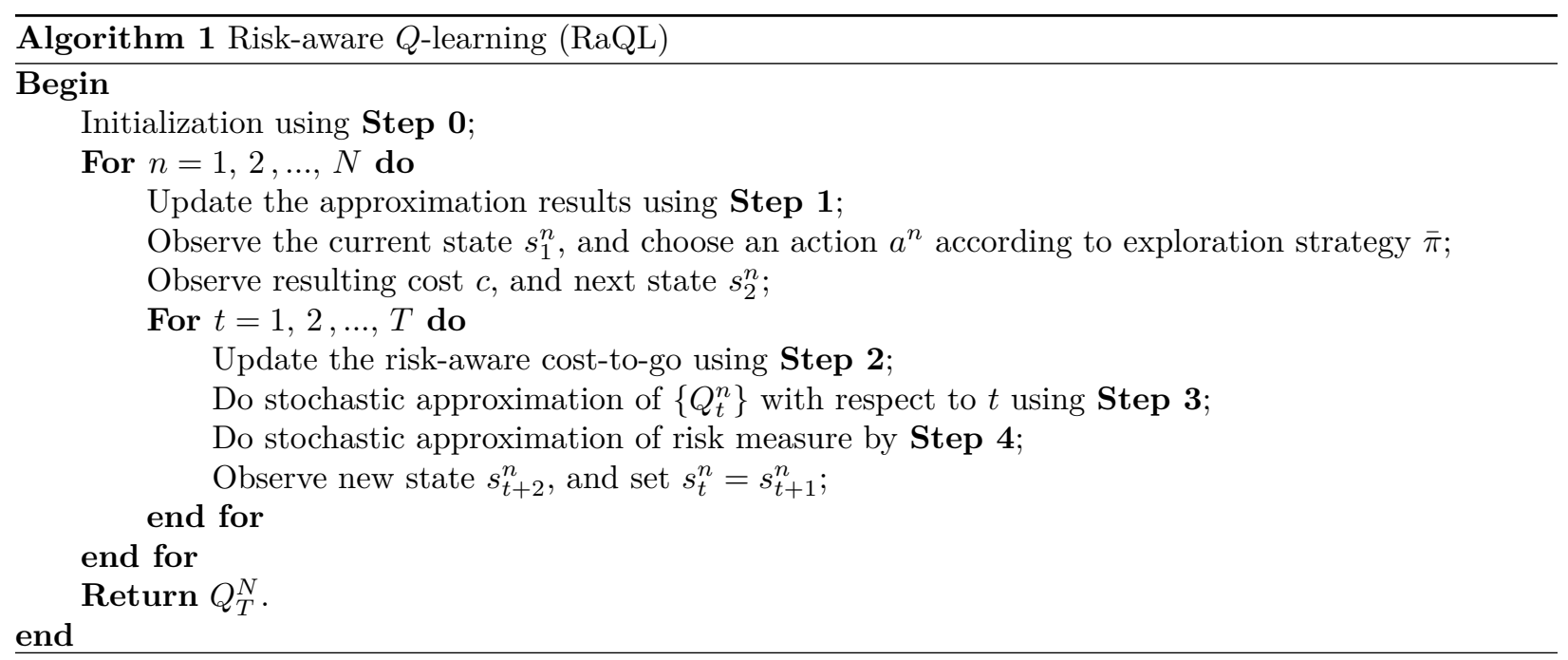

outer loop (with $N$ iterations) and an inner loop (with $T$ iterations). In Algorithm 1, we let $Q_{t}^{n}(s, a)$ be the $Q$-value of state-action pair $(s, a) \in \mathbb{K}$ w.r.t. iteration $n \leq N$ and $t \leq T$. Define $\tau_{*}(\cdot)$ to be a deterministic function with $\tau_{*}(n) \in[1, n]$ for all $n \leq N$ satisfying the same conditions as in [45, Algorithm 2.1], and define $H_{\mathcal{Y}}$ and $H_{\mathcal{Z}}$ to be any positive constants. Here we use $\left(y_{t}^{n}(s, a), z_{t}^{n}(s, a)\right)$ to denote the approximate saddle-point of Problem 4.3 for $(s, a) \in \mathbb{K}$ for all $n \leq N$ and $t \leq T$. The step-sizes are $\theta_{k}^{n}(s, a)$ (outer loop) and $\lambda_{t, \alpha}$ (inner loop), and the exploration policy is $\bar{\pi}$.

Define the collection of state-action pairs $\mathcal{G}:=\sigma\left\{\left(s_{t}^{n}, a^{n}\right), n \leq N, t \leq T\right\}$, and the filtration is $\mathcal{G}_{t}^{n}=$ $\left\{\sigma\left\{\left(s_{\tau}^{i}, a_{\tau}^{i}\right), i<n, \tau \leq T\right\} \cup\left\{\left(s_{\tau}^{n}, a_{\tau}^{n}\right), \tau \leq t\right\}\right\}$ for all $t \leq T$ and $n \leq N$, with $\mathcal{G}_{t}^{0}=\left\{\varnothing, \Omega^{\prime}\right\}$ for all $t \leq T$. This filtration is nested $\mathcal{G}_{t}^{n} \subseteq \mathcal{G}_{t+1}^{n}$ for all $1 \leq t \leq T-1$ and $\mathcal{G}_{T}^{n} \subseteq \mathcal{G}_{0}^{n+1}$, for all $1 \leq n \leq N-1$, and captures the history of the algorithm.

Definition 4.2. Given $\varepsilon \in(0,1), \bar{\pi}$ is an $\varepsilon$-greedy exploration policy that chooses a random action uniformly with probability $\varepsilon$ and otherwise (with probability $1-\varepsilon$ ) greedily chooses the action with minimal $Q$-value. We denote $a^{\prime} \in \arg \min _{a \in \mathbb{A}} Q_{T}^{n-1}(s, a)$ and suppose $\bar{\pi}$ satisfies $\mathbb{P}\left(\left(s_{t}^{n}, a^{n}\right)=(s, a) \mid \mathcal{G}_{t-1}^{n}\right)=\varepsilon$ and $\mathbb{P}\left(\left(s_{t}^{n}, a^{n}\right)=\left(s, a^{\prime}\right) \mid \mathcal{G}_{t-1}^{n}\right)=1-\varepsilon$ for all $(s, a) \in \mathbb{K}$, for any $n \leq N, t \leq T$. Similarly, we have $\mathbb{P}\left(\left(s_{1}^{n}, a^{n}\right)=(s, a) \mid \mathcal{G}_{T}^{n-1}\right)=\varepsilon$, and $\mathbb{P}\left(\left(s_{t}^{n}, a^{n}\right)=\left(s, a^{\prime}\right) \mid \mathcal{G}_{T}^{n-1}\right)=1-\varepsilon$ for all $(s, a) \in \mathbb{K}$, for any $n \leq N, t \leq$ $T$.

The exploration policy $\bar{\pi}$ in Definition 4.2 guarantees, by the Extended Borel-Cantelli Lemma in [21], that we will visit every state-action pair infinitely often with probability one. This balances exploration and exploitation in RaQL more generally, which helps the algorithm avoid getting stuck at locally optimal policies. It should be noted that RaQL is an off-policy learning algorithm, so the policy for exploration i.e. $\bar{\pi}$ and the policy from the $Q$-value updates (i.e. $\pi$ ) are different.

Assumption 4.3. For all $(s, a) \in \mathbb{K}$ and for all $n \leq N, t \leq T$, the step-sizes for the $Q$-value update satisfy: $\sum_{n=1}^{\infty} \theta_{k}^{n}(s, a)=\infty$ and $\sum_{n=1}^{\infty} \theta_{k}^{n}(s, a)^{2}<\infty$ for all $t \leq \bar{T}$ and $(s, a) \in \mathbb{K}$ a.s. Let $\#(s, a, n)$ denote one plus the number of times, until the beginning of iteration $n$, that the state-action pair $(s, a)$ has been visited, and let $N^{s, a}$ denote the set of outer iterations where action a was performed in state s. The step-sizes $\theta_{k}^{n}(s, a)$ satisfy $\theta_{k}^{n}(s, a):=\frac{1}{[\#(s, a, n)]^{k}}$ if $n \in N^{s, a}$ and $\theta_{k}^{n}(s, a)=0$ otherwise.

Assumption 4.3 sends the step-size to zero whenever a state-action pair is not visited. This step-size selection ensures that only a single state-action pair is updated when it is observed, which reveals the asynchronous nature of the $Q$-learning algorithm stated in $[26$. We choose $k \in(1 / 2,1]$, where we call $k=1$ a linear learning rate and $k \in(1 / 2,1)$ a polynomial learning rate, and step-sizes $\lambda_{t, \alpha}=C t^{-\alpha}$ for the risk estimation with $\alpha \in(0,1]$ for arbitrary $C>0$. The detailed description for each step of RaQL follows:

Step 0: Initialize an approximation for the $Q$-values $Q^{0}(s, a)$ for all $(s, a) \in \mathbb{K}$; given step-sizes $\theta_{k}^{n}, \lambda_{t, \alpha}>$ 0 for $t \leq T$ and $n \leq N$, with learning rates $k$ and $\alpha$; deterministic function $\tau^{*}(\cdot)$; initialize $\left(y_{t}^{0}(s, a), z_{t}^{0}(s, a)\right)$ for all $t \leq T$ and $(s, a) \in \mathbb{K}$. 
Step 1: For all $(s, a) \in \mathbb{K}$, set $\left(y_{1}^{n}(s, a), z_{1}^{n}(s, a)\right)=\left(y_{T}^{n-1}(s, a), z_{T}^{n-1}(s, a)\right)$ and $Q_{1}^{n}(s, a)=Q_{T}^{n-1}(s, a)$.

Step 2: Compute $v^{n-1}\left(s_{t+1}^{n}\right)=\min _{a \in \mathbb{A}} Q_{T}^{n-1}\left(s_{t+1}^{n}, a\right)$. Compute

$$
\hat{q}_{t}^{n}\left(s_{t}^{n}, a^{n}\right)=c\left(s_{t}^{n}, a^{n}\right)+\gamma G\left(v^{n-1}\left(s_{t+1}^{n}\right), y^{n, t}\left(s_{t}^{n}, a^{n}\right), z^{n, t}\left(s_{t}^{n}, a^{n}\right)\right),
$$

and

$$
\left(y^{n, t}\left(s_{t}^{n}, a^{n}\right), z^{n, t}\left(s_{t}^{n}, a^{n}\right)\right)=\frac{1}{t-\tau_{*}(t)+1} \sum_{\tau=\tau_{*}(t)}^{t}\left(y_{\tau}^{n}\left(s_{t}^{n}, a^{n}\right), z_{\tau}^{n}\left(s_{t}^{n}, a^{n}\right)\right) .
$$

To explain, given iteration $n$, in each iteration $t \leq T$, we observe a new state $s_{t+1}^{n}$ given current state $s_{t}^{n}$ and action $a^{n}$, compute the estimated risk-aware cost-to-go $\hat{q}_{t}^{n}$ from one sample in Eq. (4.7). Here, we use the $Q$-value $Q_{T}^{n-1}$ at iteration $T$ and we compute $v^{n-1}$ from it as input for Eq. (4.7), although all the $Q$-values $\left\{Q_{t}^{n-1}\right\}_{t=1}^{T}$ are recorded.

Step 3: For all $(s, a) \in \mathbb{K}$, compute

$$
Q_{t}^{n}(s, a)=\left(1-\theta_{k}^{n}(s, a)\right) Q_{T}^{n-1}(s, a)+\theta_{k}^{n}(s, a) \hat{q}_{t}^{n}\left(s_{t}^{n}, a^{n}\right) .
$$

This update is the same as in standard $Q$-learning w.r.t. the outer loop.

Step 4: Update

$$
\begin{aligned}
\left(y_{t+1}^{n}\left(s_{t}^{n}, a^{n}\right), z_{t+1}^{n}\left(s_{t}^{n}, a^{n}\right)\right)= & \Pi_{\mathcal{Y} \times \mathcal{Z}}\left\{\left(y_{t}^{n}\left(s_{t}^{n}, a^{n}\right), z_{t}^{n}\left(s_{t}^{n}, a^{n}\right)\right)\right. \\
& \left.-\lambda_{t, \alpha} \psi\left(v^{n-1}\left(s_{t+1}^{n}\right), y^{n, t}\left(s_{t}^{n}, a^{n}\right), z^{n, t}\left(s_{t}^{n}, a^{n}\right)\right)\right\},
\end{aligned}
$$

where $\Pi_{\mathcal{Y} \times \mathcal{Z}}[(y, z)]:=\arg \min _{\left(y^{\prime}, z^{\prime}\right) \in \mathcal{Y} \times \mathcal{Z}}\left\|(y, z)-\left(y^{\prime}, z^{\prime}\right)\right\|_{2}$ is the Euclidean projection onto $\mathcal{Y} \times \mathcal{Z}$, and

$$
\psi\left(v^{n-1}\left(s_{t+1}^{n}\right), y^{n, t}\left(s_{t}^{n}, a^{n}\right), z^{n, t}\left(s_{t}^{n}, a^{n}\right)\right)=\left(\begin{array}{c}
H_{\mathcal{Y}} G_{y}\left(v^{n-1}\left(s_{t+1}^{n}\right), y^{n, t}\left(s_{t}^{n}, a^{n}\right), z^{n, t}\left(s_{t}^{n}, a^{n}\right)\right) \\
-H_{\mathcal{Z}} G_{z}\left(v^{n-1}\left(s_{t+1}^{n}\right), y^{n, t}\left(s_{t}^{n}, a^{n}\right), z^{n, t}\left(s_{t}^{n}, a^{n}\right)\right)
\end{array}\right) .
$$

We provide some further remarks on Algorithm 1.

1. In Eqs. 4.8), 4.10), and 4.11, we use the stochastic approximation for saddle-point problems (SASP) algorithm as presented in [45, Algorithm 2.1] (the detailed steps appear in Algorithm 2). In Algorithm 1 , we apply and extend SASP to estimate the risk with respect to each state-action pair, where the value functions on random next states are the problem input. Classic stochastic approximation may result in extremely slow convergence for degenerate objectives (i.e. the objective has a singular Hessian). However, based on the analysis in [45], SASP with properly chosen $\alpha \in(0,1]$ preserves a "reasonable" (close to $O\left(n^{-1 / 2}\right)$ ) convergence rate even when the objective is non-smooth and/or degenerate. For instance, the Kusuoka representation (3.5) is non-smooth and degenerate since the Hessian matrix is singular with respect to $p \in \mathfrak{M}$. Thus, SASP is more appropriate for estimation of risk measures.

2. The risk estimation and the $Q$-value updates are mutually dependent. Given iteration $n$, the risk estimation, Step 4, applies SASP to update the candidate solution of the saddle-point problem for each selected state-action pair, using the $Q$-value from the previous iteration (i.e. $Q_{T}^{n-1}$ ). Given the current state-action pair $(s, a)$, neither the expected value of $G$ in $(4.3)$, nor the subdifferentials $\left\{\partial_{y} \mathbb{E} G\left(v\left(s^{\prime}\right), y, z\right), \partial_{z} \mathbb{E} G\left(v\left(s^{\prime}\right), y, z\right)\right\}$ (the expectation is with respect to the transition kernel), are available. We assume that at any iteration $t$, for every desired point $\left(y^{n, t}\left(s_{t}^{n}, a^{n}\right), z^{n, t}\left(s_{t}^{n}, a^{n}\right)\right)$, one can obtain a biased estimator of the aforementioned subgradients. These estimates form a realization of the pair of random vectors,

$$
G_{y}\left(v^{n-1}\left(s_{t+1}^{n}\right), y^{n, t}\left(s_{t}^{n}, a^{n}\right), z^{n, t}\left(s_{t}^{n}, a^{n}\right)\right) \in \mathbb{R}^{d_{1}},
$$

and

$$
G_{z}\left(v^{n-1}\left(s_{t+1}^{n}\right), y^{n, t}\left(s_{t}^{n}, a^{n}\right), z^{n, t}\left(s_{t}^{n}, a^{n}\right)\right) \in \mathbb{R}^{d_{2}},
$$

where $\left\{v^{n-1}\left(s_{t}^{n}\right)\right\}_{t=1}^{T}$ is a sequence of independent identically distributed "observation noises" according to the underlying transition kernel. In Step 3, the $Q$-value in the current iteration $\left\{Q_{t}^{n}\right\}_{t=1}^{T}$ is updated based on $Q_{T}^{n-1}$ and the approximate risk-to-go $\hat{q}_{t}^{n}\left(s_{t}^{n}, a^{n}\right)$ follows the same update rule in standard $Q$-learning. 
Algorithm 2 SASP

Step 0. Input: i.i.d samples $\left\{x_{t}\right\}_{t=1}^{\infty}$ of random variable $X$; step-sizes $\lambda_{t}=C t^{-\alpha}$ with $\alpha \in(0,1]$ for $C>0$; deterministic function $\tau_{*}(\cdot)$; initial $\left(y_{1}, z_{1}\right) \in \mathcal{Y} \times \mathcal{Z}$; positive constants $H_{\mathcal{Y}}, H_{\mathcal{Z}}$,

Step 1. for $t=1,2, \ldots$ do

Step 1a. Update

$$
\left(y_{t+1}, z_{t+1}\right)=\Pi_{\mathcal{Y} \times \mathcal{Z}}\left[\left(y_{t}, z_{t}\right)-\lambda_{n} \psi\left(x_{t} ; y_{t}, z_{t}\right)\right], t \geq 1 .
$$

The vector $\psi(x ; y, z) \in \mathbb{R}^{d_{1}} \times \mathbb{R}^{d_{2}}$ is

$$
\psi(x ; y, z)=\left(H_{\mathcal{Y}} G_{y}(x, y, z),-H_{\mathcal{Z}} G_{z}(x, y, z)\right),
$$

and $x$ is any realization of the random variable $X$,

Step 1b. Take the moving average

$$
\left(y_{t}, z_{t}\right)=\frac{1}{t-\tau_{*}(t)+1} \sum_{\tau=\tau_{*}(t)}^{t}\left(y_{\tau}, z_{\tau}\right) .
$$

3. We resolve the "overestimation" problem (the accumulated error from poor risk estimation) in reinforcement learning described in 32,70 through the special inner-outer loop structure of RaQL. This phenomenon is not mentioned or resolved in [66], where the iterative procedure is analogous to standard $Q$-learning because of the special structure of utility-based shortfall. RaQL reduces the bias by multiple iterations of inner loop to provide an accurate risk estimate before updating the $Q$-values. Our algorithm is thus related to "Repeated updated $Q$-learning" as proposed in [1], which resolves performance degradation when the algorithm is used in noisy non-stationary environments. Our algorithm addresses what we refer to as the "policy-bias" of the action value update. Policy-bias appears in $Q$ learning because the value of an action is only updated when the action is executed. Consequently, the effective rate of updating an action value directly depends on the probability of choosing the action for execution. For any state-action pair $\left(s_{1}^{n}, a^{n}\right)$ chosen by $\bar{\pi}$ in the outer loop w.r.t. $n \leq N$, we perform stochastic approximation to estimate the risk for state-action pairs with fixed action $a^{n}$ in iterations $t \leq T$. This convention increases the probability of choosing optimal actions while also getting a more accurate risk estimate.

4. Often, the cost function is random rather than deterministic. For example, in inventory control, for stock $s$, we order quantity $a$, and then only learn the cost after seeing the random demand. Let $c(s, a, X)$ denote the random cost, where $X$ is random noise, and assume that $0 \leq c(s, a, X) \leq C_{\max }$ for all $(s, a) \in \mathbb{K}$ a.s. Following the same technique as for standard $Q$-learning from [52,69], we can substitute realizations of the random cost for deterministic costs in our update rule Eq. (4.6), and then compute the risk of the sum of the random cost and the discounted cost-to-go,

$$
Q^{*}(s, a)=\mathcal{R}_{(s, a)}^{G}\left[c(s, a, X)+\gamma \min _{a \in \mathbb{A}} Q^{*}\left(s^{\prime}, a\right)\right],
$$

for all $(s, a) \in \mathbb{K}$. Let $\left\{x_{t}^{n}\right\}_{1 \leq t \leq T, 1 \leq n \leq N}$ denote a sequence of independent identically distributed samples of $X$ indexed by $t$ and $n$, and let $c\left(s_{t}^{n}, a^{n}, x_{t}^{n}\right)$ denote the cost observed in state $s_{t}^{n}$, for action $a^{n}$, with noise $x_{t}^{n}$ at iteration $t$ and $n$. In terms of solving Problem 4.12, we replace the earlier expression (4.7) in Algorithm 1 with

$$
\hat{q}_{t}^{n}\left(s_{t}^{n}, a^{n}\right)=G\left(c\left(s_{t}^{n}, a^{n}, x_{t}^{n}\right)+\gamma v^{n-1}\left(s_{t+1}^{n}\right), y^{n, t}\left(s_{t}^{n}, a^{n}\right), z^{n, t}\left(s_{t}^{n}, a^{n}\right)\right),
$$

and replace Step 4 in Algorithm 1 with

$$
\begin{aligned}
\left(y_{t+1}^{n}\left(s_{t}^{n}, a^{n}\right), z_{t+1}^{n}\left(s_{t}^{n}, a^{n}\right)\right)= & \Pi_{\mathcal{Y} \times \mathcal{Z}}\left\{\left(y_{t}^{n}\left(s_{t}^{n}, a^{n}\right), z_{t}^{n}\left(s_{t}^{n}, a^{n}\right)\right)\right. \\
& \left.-\lambda_{t, \alpha} \psi\left(c\left(s_{t}^{n}, a^{n}, x_{t}^{n}\right)+\gamma v^{n-1}\left(s_{t+1}^{n}\right), y^{n, t}\left(s_{t}^{n}, a^{n}\right), z^{n, t}\left(s_{t}^{n}, a^{n}\right)\right)\right\},
\end{aligned}
$$


and

$$
\begin{aligned}
& \psi\left(v^{n-1}\left(s_{t+1}^{n}\right), y^{n, t}\left(s_{t}^{n}, a^{n}\right), z^{n, t}\left(s_{t}^{n}, a^{n}\right)\right) \\
= & \left(\begin{array}{c}
H_{\mathcal{Y}} G_{y}\left(c\left(s_{t}^{n}, a^{n}, x_{t}^{n}\right)+\gamma v^{n-1}\left(s_{t+1}^{n}\right), y^{n, t}\left(s_{t}^{n}, a^{n}\right), z^{n, t}\left(s_{t}^{n}, a^{n}\right)\right) \\
-H_{\mathcal{Z}} G_{z}\left(c\left(s_{t}^{n}, a^{n}, x_{t}^{n}\right)+\gamma v^{n-1}\left(s_{t+1}^{n}\right), y^{n, t}\left(s_{t}^{n}, a^{n}\right), z^{n, t}\left(s_{t}^{n}, a^{n}\right)\right)
\end{array}\right) .
\end{aligned}
$$

This random cost variant of Algorithm 1 is also based on repeated stochastic approximation for risk estimation for a fixed action in the inner loop, which resolves the overestimation problem caused by the biased risk estimation.

\subsection{Main results}

We now state our convergence results for RaQL.

Theorem 4.4. (Almost Sure Convergence) Suppose Assumption 4.3 holds, and fix $T \geq 1$. Let $\left\{Q_{T}^{n}\right\}_{n \geq 1}$ be the $Q$-value produced by Algorithm 1. Then $Q_{T}^{n} \rightarrow Q^{*}$ as $n \rightarrow \infty$, almost surely.

The proof of Theorem 4.4 uses techniques from the stochastic approximation literature [40], [19] and [18, which are applied to reinforcement learning and $Q$-learning in [14, 35, 69,71. However, our algorithm differs from risk-neutral $Q$-learning because it updates $Q$-values as well as estimates risk via stochastic approximation. The intuition of our proof follows the idea in [36] where multiple "stochastic approximation instances" for both $Q$-value updates and risk estimation are "pasted" together. The error in $Q$-values is captured by the distance of $Q_{t}^{n}$ to the optimal $Q^{*}$, while the error in risk estimation is captured by the duality gap of the corresponding stochastic saddle-point problem. We must account for the interdependence of these two errors in several parts of our proof.

Next, we present the convergence rate for RaQL for a polynomial learning rate. We first clarify several important concepts and definitions that appear in this result. For any $(s, a) \in \mathbb{K}$, we define $\left(y^{n, *}(s, a), z^{n, *}(s, a)\right)$ to be a saddle-point of

$$
(y(s, a), z(s, a)) \rightarrow \mathbb{E}_{s^{\prime} \sim P(\cdot \mid s, a)}\left[G\left(v^{n-1}\left(s^{\prime}\right), y(s, a), z(s, a)\right)\right],
$$

for each $(s, a) \in \mathbb{K}$, where $v^{n-1}\left(s^{\prime}\right):=\min _{a \in \mathbb{A}} Q_{T}^{n}\left(s^{\prime}, a\right)$. Similarly, we define $\left(y^{*}(s, a), z^{*}(s, a)\right)$ to be a saddle-point of

$$
(y(s, a), z(s, a)) \rightarrow \mathbb{E}_{s^{\prime} \sim P(\cdot \mid s, a)}\left[G\left(v^{*}\left(s^{\prime}\right), y(s, a), z(s, a)\right)\right],
$$

for each $(s, a) \in \mathbb{K}$, where $v^{*}\left(s^{\prime}\right):=\min _{a \in \mathbb{A}} Q^{*}\left(s^{\prime}, a\right)$. We define the Hausdorff distance between sets with respect to the Euclidean norm based on [54. Let $\mathfrak{A}$ and $\mathfrak{B}$ be two non-empty subsets of a metric space $\left(M,\|\cdot\|_{2}\right)$. We define their Hausdorff distance $\mathfrak{D}_{H}(\mathfrak{A}, \mathfrak{B})$ by

$$
\mathfrak{D}_{H}(\mathfrak{A}, \mathfrak{B}):=\max \left\{\sup _{A \in \mathfrak{A}} \inf _{B \in \mathfrak{B}}\|A-B\|_{2}, \sup _{B \in \mathfrak{B}} \inf _{A \in \mathfrak{A}}\|A-B\|_{2}\right\}
$$

Let

$$
\mathcal{S}_{1}^{n, t}:=\left\{\left(\partial G_{y}\left(v^{n-1}, y^{n, t}, z^{n, t}\right), \partial G_{z}\left(v^{n-1}, y^{n, t}, z^{n, t}\right)\right)\right\}
$$

and

$$
\mathcal{S}_{2}^{n, t}:=\left\{\left(\partial G_{y}\left(v^{*}, y^{n, t}, z^{n, t}\right), \partial G_{z}\left(v^{*}, y^{n, t}, z^{n, t}\right)\right)\right\},
$$

be the two subdifferentials of $G$ with respect to $v^{n-1}$ and $v^{*}$, given $\left(y^{n, t}, z^{n, t}\right)$. The results of the following lemmas appear in our main convergence rate result. First, Lemma 4.5 bounds $\mathfrak{D}_{H}\left(\mathcal{S}_{1}^{n, t}, \mathcal{S}_{2}^{n, t}\right)$ with respect to $\left\|Q_{T}^{n-1}-Q^{*}\right\|_{2}$.

Lemma 4.5. [5] [49, Theorem 4.1] Suppose Assumption 3.1 (ii) holds, then there exist $K_{\psi}^{(1)}, K_{\psi}^{(2)}>0$, such that

$$
\mathfrak{D}_{H}\left(\mathcal{S}_{1}^{n, t}, \mathcal{S}_{2}^{n, t}\right) \leq K_{\psi}^{(1)}\left\|Q_{T}^{n-1}-Q^{*}\right\|_{2}+K_{\psi}^{(2)} \sqrt{\left\|Q_{T}^{n-1}-Q^{*}\right\|_{2}}
$$

for all $n \leq N$ and $t \leq T$. 
The next result pertains to the modulus of stability of the saddle-points for the estimated risk measure w.r.t. each $n \leq N$.

Lemma 4.6. [68, Theorem 3.1] [41, Proposition 3.1] Suppose Assumption 3.1 holds, then there exists $K_{S}>0$ such that for all $n \leq N$ we have

$$
\begin{aligned}
& \left\|\left(y^{*}, z^{*}\right)-\left(y^{n, *}, z^{n, *}\right)\right\|_{2} \\
\leq & K_{S} \| \mathbb{E}_{s^{\prime} \sim P(\cdot \mid s, a)}\left[G\left(v^{n-1}\left(s^{\prime}\right), y^{*}(s, a), z^{*}(s, a)\right)\right] \\
& -\mathbb{E}_{s^{\prime} \sim P(\cdot \mid s, a)}\left[G\left(v^{n-1}\left(s^{\prime}\right), y^{n, *}(s, a), z^{n, *}(s, a)\right)\right] \|_{2} .
\end{aligned}
$$

Theorem 4.7. (High Probability Convergence Rate) Suppose Assumption 4.3 holds, and choose $\tilde{\varepsilon}>0$ and $\delta \in(0,1)$. For a polynomial learning rate (i.e., $k \in(1 / 2,1)$ ), there exist $0<\kappa<1 / C K_{\psi}^{(1)}$ and

$$
\beta_{T}:=\frac{K_{G}}{2}\left\{1-\gamma-\sqrt{\frac{C\left(\tau_{*}(T)\right)^{-\alpha}}{\kappa\left(1-C\left(\tau_{*}(T)\right)^{-\alpha} K_{\psi}^{(1)} \kappa\right)}}-K_{G} K_{S}\right\},
$$

such that we have $\left\|Q_{T}^{N}-Q^{*}\right\|_{2} \leq \tilde{\varepsilon}$ with probability at least $1-\delta$, for $N$ and $T$ satisfying:

$$
\begin{gathered}
\left(\tau_{*}(T)\right)^{-\alpha} \leq \frac{\left(1-\gamma-K_{G} K_{S}\right)^{2} \kappa / C}{1+K_{\psi}^{(1)}\left(1-\gamma-K_{G} K_{S}\right)^{2} \kappa^{2}}, \\
\left(\tau_{*}(T)\right)^{-\alpha} \geq \frac{\left[K_{G}-K_{G}\left(2 \gamma+K_{G} K_{S}\right)-2\right]^{2} \kappa}{C\left\{K_{G}+\left[K_{G}-K_{G}\left(2 \gamma+K_{G} K_{S}\right)-2\right]^{2} K_{\psi}^{(1)} \kappa^{2}\right\}},
\end{gathered}
$$

and

$$
N=\Omega\left(\left(\frac{V_{\max }^{2}|\mathbb{S}||\mathbb{A}| \ln \left(V_{\max }(|\mathbb{S}||\mathbb{A}|)^{3 / 2} /\left[\delta \beta_{T} \tilde{\varepsilon}(1-\varepsilon)\right]\right.}{\beta_{T}^{2} \tilde{\varepsilon}^{2}(1-\varepsilon)^{1+3 k}}\right)^{1 / k}+\left(\frac{1}{(1-\varepsilon) \beta_{T}} \ln \frac{V_{\max } \sqrt{|\mathbb{S}||\mathbb{A}|}}{\tilde{\varepsilon}}\right)^{\frac{1}{1-k}}\right)
$$

Remark 4.8. To interpret the bound (4.18), we first consider its dependence on $\tilde{\varepsilon}$. This dependence gives us the bound $\Omega\left(\left(\ln (1 / \tilde{\varepsilon}) / \tilde{\varepsilon}^{2}\right)^{1 / k}+(\ln (1 / \tilde{\varepsilon}))^{1 /(1-k)}\right)$, which mirrors the bound for classical asynchronous $Q$ learning in [26. Theorem 4]. The lower bound on the number of outer iterations $N$ (4.18) is decreasing with $\beta_{T}$. Since the quantity $\beta_{T}$ is increasing with $T$, the lower bound on $N$ is decreasing with $T$. Consequently, improving the quality of risk estimation by increasing the number of inner loops will improve the overall convergence rate of the algorithm. In addition, the sample complexity will first decrease and then increase as a function of the learning rate $k$ (which is also observed for standard $Q$-learning in [26]). Furthermore, the sample complexity is directly proportional to the discount factor $\gamma$, problem size $|\mathbb{S}||\mathbb{A}|$, and bound $V_{\max }$ on the magnitude of the value functions in $\mathcal{V}$. It is inversely proportional to the Lipschitz constant $K_{G}$ and the modulus of the Hausdorff distance of the subdifferentials $K_{\psi}^{(1)}$. Increasing $\varepsilon$ will also increase the sample complexity, revealing that there is a tradeoff between avoiding the algorithm getting stuck at local optima and reducing the overall computational complexity. The sample complexity also depends on the risk measure, since different risk measures have different constants $K_{G}, K_{\psi}^{(1)}$, and $K_{S}$.

We also derive the convergence rate of RaQL for a linear learning rate (i.e., $k=1$ ) in Theorem 4.9 Under a linear learning rate, we can obtain convergence rate results in both probability and expectation as summarized in Theorem 4.10

Theorem 4.9. (High Probability Convergence Rate) Suppose Assumption 4.3 holds, and choose $\tilde{\varepsilon}>0$ and $\delta \in(0,1)$. For a linear learning rate $k=1$, there exists $\beta_{T}$ as described in 4.15), such that we have $\left\|Q_{T}^{N}-Q^{*}\right\|_{2} \leq \tilde{\varepsilon}$ with probability at least $1-\delta$, for $N$ satisfying

$$
N=\Omega\left(\left(\frac{2+\Psi-\varepsilon}{1-\varepsilon}\right)^{\frac{1}{\beta_{T}} \ln \frac{V_{\max } \sqrt{|\mathbb{S}||\mathbb{A}|}}{\tilde{\varepsilon}}} \frac{V_{\max }^{2}|\mathbb{S}||\mathbb{A}| \ln \left(V_{\max }(|\mathbb{S}||\mathbb{A}|)^{3 / 2} /\left[\Psi \delta \beta_{T} \tilde{\varepsilon}(1-\varepsilon)\right]\right)}{\Psi^{2} \beta_{T} \tilde{\varepsilon}^{2}(1-\varepsilon)^{2}}\right),
$$

where $\Psi$ is any positive constant, and for $T$ satisfying conditions 4.16) and 4.17). 
In Theorem 4.9 the positive constant $\Psi$ is used to bound the duration of iteration $m$, which starts at time $\tau_{m}$, and ends at time $\tau_{m+1}$. Define $C_{G}$ be the upper bound:

$$
\left\{1+\frac{C}{\kappa\left(1-C K_{\psi}^{(1)} \kappa\right)}+\left[K_{G}\left(\gamma+K_{S} K_{G}\right)\right]^{2}\right\}\left\|Q_{T}^{n-1}-Q^{*}\right\|_{2}^{2} \leq C_{G},
$$

almost surely. Next, we prove convergence in expectation. We define a function

$$
\begin{aligned}
f(t):= & {\left[K_{\mathcal{Y}} H_{\mathcal{Y}}^{-1}+K_{\mathcal{Z}} H_{\mathcal{Z}}^{-1}\right] \frac{t^{\alpha}}{C\left(t-\tau_{*}(t)+1\right)} } \\
& +\frac{\left(K_{\mathcal{Y}}+K_{\mathcal{Z}}\right) L}{\sqrt{t-\tau_{*}(t)+1}} \\
& +C\left(K_{\mathcal{Y}}+K_{\mathcal{Z}}\right)^{2} L^{2}\left[H_{\mathcal{Y}} K_{\mathcal{Y}}+H_{\mathcal{Z}} K_{\mathcal{Z}}\right] \tau_{*}^{-\alpha}(t),
\end{aligned}
$$

for all integers $1 \leq t \leq T$.

Theorem 4.10. (Convergence Rate in Expectation) Suppose Assumption 4.3 holds, and set linear learning rate $k=1$. Given $\tilde{\varepsilon}>0$, we have $\mathbb{E}\left[\left\|Q_{T}^{N}-Q^{*}\right\|_{2} \mid \mathcal{G}_{T+1}^{N-1}\right] \leq \tilde{\varepsilon}$, for $N$ satisfying

$$
N=\Omega\left(\frac{\max \left\{\left(C_{G}+(\gamma f(T))^{2}\right) \varepsilon /\left(\left(2-2 \gamma K_{G}\right) \varepsilon^{2}-K_{G}\left(\gamma-K_{S} K_{G}-\varepsilon\right), C_{\max }^{2}|\mathbb{S}||\mathbb{A}|\right\}\right.}{\tilde{\varepsilon}}\right),
$$

where $C_{G}$ is defined in the inequality (4.19) and $f(T)$ is defined in Eq. (4.20) by choosing $t=T$.

In Theorem 4.10, the function $f(t)$ bounds the duality gap of the stochastic saddle-point estimation in each iteration $t \leq T$, for a fixed iteration $n \leq N$.

\section{Proofs of main results}

\subsection{Almost sure convergence}

We now present the proof of Theorem 4.4 step by step.

Step 1: Bounding $\left\|\left(y^{n, t}, z^{n, t}\right)-\left(y^{n, *}, z^{n, *}\right)\right\|_{2}^{2}$, for all $n \leq N$ and $t \leq T$, by a function of $\left\|Q_{T}^{n-1}-Q^{*}\right\|_{2}^{2}$.

Lemma 5.1. Suppose Assumption 4.3 holds, then there exists $0<\kappa<1 /\left(C K_{\psi}^{(1)}\right)$ such that

$$
\left\|\left(y^{n, t}, z^{n, t}\right)-\left(y^{n, *}, z^{n, *}\right)\right\|_{2}^{2} \leq \frac{C\left(\tau_{*}(t)\right)^{-\alpha}}{\kappa\left(1-C\left(\tau_{*}(t)\right)^{-\alpha} K_{\psi}^{(1)} \kappa\right)}\left\|Q_{T}^{n-1}-Q^{*}\right\|_{2}^{2},
$$

for all $t \leq T, n \leq N$.

Proof. From Eq. 4.10 in Step 4 of Algorithm 1, we know

$$
\begin{aligned}
\left\|\left(y_{t+1}^{n}, z_{t+1}^{n}\right)-\left(y^{n, *}, z^{n, *}\right)\right\|_{2}^{2}= & \left\|\prod_{\mathcal{Y} \times \mathcal{Z}}\left(\left(y_{t}^{n}, z_{t}^{n}\right)-\lambda_{t, \alpha} \psi\left(v^{n-1}, y^{n, t}, z^{n, t}\right)\right)-\prod_{\mathcal{Y} \times \mathcal{Z}}\left(y^{n, *}, z^{n, *}\right)\right\|_{2}^{2} \\
\leq & \left\|\left(y_{t}^{n}, z_{t}^{n}\right)-\left(y^{n, *}, z^{n, *}\right)-\lambda_{t, \alpha} \psi\left(v^{n-1}, y^{n, t}, z^{n, t}\right)\right\|_{2}^{2} \\
\leq & \left\|\left(y_{t}^{n}, z_{t}^{n}\right)-\left(y^{n, *}, z^{n, *}\right)\right\|_{2}^{2}+\left(H_{\mathcal{Y}}^{2}+H_{\mathcal{Z}}^{2}\right) L^{2} C^{2} t^{-2 \alpha} \\
& -2 C t^{-\alpha}\left(\left(y_{t}^{n}, z_{t}^{n}\right)-\left(y^{n, *}, z^{n, *}\right)\right)^{\top} \psi\left(v^{n-1}, y^{n, t}, z^{n, t}\right),
\end{aligned}
$$

where the first inequality follows from non-expansiveness of the projection operator and the second inequality holds by Assumption 3.1(iv). Based on Lemma 4.5, we have

$$
\left\|\psi\left(v^{n-1}, y^{n, t}, z^{n, t}\right)-\psi\left(v^{*}, y^{n, t}, z^{n, t}\right)\right\|_{2} \leq \mathfrak{D}\left(\mathcal{S}_{1}^{n, t}, \mathcal{S}_{2}^{n, t}\right) \leq K_{\psi}^{(1)}\left\|Q_{T}^{n-1}-Q^{*}\right\|_{2}+K_{\psi}^{(2)} \sqrt{\left\|Q_{T}^{n-1}-Q^{*}\right\|_{2}} .
$$


Take the sum of the terms $C t^{-\alpha}\left(\left(y_{t}^{n}, z_{t}^{n}\right)-\left(y^{n, *}, z^{n, *}\right)\right)^{\top} \psi\left(v^{n-1}, y^{n, t}, z^{n, t}\right)$ from $\tau_{*}(t)$ to $t$, divide by $\frac{1}{t-\tau_{*}(t)+1}$, to obtain:

$$
\begin{aligned}
& \frac{1}{t-\tau_{*}(t)+1} \sum_{\tau=\tau_{*}(t)}^{t}\left[C \tau^{-\alpha}\left(\left(y_{\tau}^{n}, z_{\tau}^{n}\right)-\left(y^{n, *}, z^{n, *}\right)\right)^{\top} \psi\left(v^{n-1}, y^{n, t}, z^{n, t}\right)\right] \\
\leq & C\left(\tau_{*}(t)\right)^{-\alpha}\left(\left(y^{n, t}, z^{n, t}\right)-\left(y^{n, *}, z^{n, *}\right)\right)^{\top}\left(\psi\left(v^{n-1}, y^{n, t}, z^{n, t}\right)-\psi\left(v^{*}, y^{n, t}, z^{n, t}\right)\right) \\
\leq & C\left(\tau_{*}(t)\right)^{-\alpha}\left\|\left(y^{n, t}, z^{n, t}\right)-\left(y^{n, *}, z^{n, *}\right)\right\|_{2}\left\|\psi\left(v^{n-1}, y^{n, t}, z^{n, t}\right)-\psi\left(v^{*}, y^{n, t}, z^{n, t}\right)\right\|_{2} \\
\leq & C\left(\tau_{*}(t)\right)^{-\alpha}\left\|\left(y^{n, t}, z^{n, t}\right)-\left(y^{n, *}, z^{n, *}\right)\right\|_{2}\left(K_{\psi}^{(1)}\left\|Q_{T}^{n-1}-Q^{*}\right\|_{2}+K_{\psi}^{(2)} \sqrt{\left\|Q_{T}^{n-1}-Q^{*}\right\|_{2}}\right),
\end{aligned}
$$

where the first inequality holds due to Assumption 3.1(iii). Using the inequality $2 a b \leq a^{2} \kappa+b^{2} / \kappa$ for all $\kappa>0$, we obtain

$$
\begin{aligned}
& -2 C\left(\tau_{*}(t)\right)^{-\alpha}\left(\left(y^{n, t}, z^{n, t}\right)-\left(y^{n, *}, z^{n, *}\right)\right)^{\top}\left(\psi\left(v^{n-1}, y^{n, t}, z^{n, t}\right)-\psi\left(v^{*}, y^{n, t}, z^{n, t}\right)\right) \\
\geq & -C\left(\tau_{*}(t)\right)^{-\alpha} K_{\psi}^{(1)}\left\|\left(y^{n, t}, z^{n, t}\right)-\left(y^{n, *}, z^{n, *}\right)\right\|_{2}^{2} \kappa-C\left(\tau_{*}(t)\right)^{-\alpha}\left\|Q_{T}^{n-1}-Q^{*}\right\|_{2}^{2} / \kappa \\
- & C\left(\tau_{*}(t)\right)^{-\alpha} K_{\psi}^{(2)}\left\|\left(y^{n, t}, z^{n, t}\right)-\left(y^{n, *}, z^{n, *}\right)\right\|_{2} \sqrt{\left\|Q_{T}^{n-1}-Q^{*}\right\|_{2}} .
\end{aligned}
$$

By summing the right hand side of 5.2 from $\tau_{*}(t)$ to $t$, dividing by $\frac{1}{t-\tau_{*}(t)+1}$, and combining with 5.3 we obtain

$$
\begin{aligned}
& \frac{1}{t-\tau_{*}(t)+1} \sum_{\tau=\tau_{*}(t)}^{t}\left(\left\|\left(y_{\tau}^{n}, z_{\tau}^{n}\right)-\left(y^{n, *}, z^{n, *}\right)\right\|_{2}^{2}+\left(H_{\mathcal{Y}}^{2}+H_{\mathcal{Z}}^{2}\right) L^{2} C^{2} \tau^{-2 \alpha}\right) \\
& -2 C\left(\tau_{*}(t)\right)^{-\alpha}\left(\left(y^{n, t}, z^{n, t}\right)-\left(y^{n, *}, z^{n, *}\right)\right)^{\top} \psi\left(v^{n-1}, y^{n, t}, z^{n, t}\right) \\
\geq & C\left(\tau_{*}(t)\right)^{-\alpha}\left\|\left(y^{n, t}, z^{n, t}\right)-\left(y^{n, *}, z^{n, *}\right)\right\|_{2}^{2}-2\left(\left(y^{n, t}, z^{n, t}\right)-\left(y^{n, *}, z^{n, *}\right)\right)^{\top} \psi\left(v^{n-1}, y^{n, t}, z^{n, t}\right) \\
\geq & \left(1-C\left(\tau_{*}(t)\right)^{-\alpha} K_{\psi}^{(1)} \kappa\right)\left\|\left(y^{n, t}, z^{n, t}\right)-\left(y^{n, *}, z^{n, *}\right)\right\|_{2}^{2} \\
& -C\left(\tau_{*}(t)\right)^{-\alpha}\left\|Q_{T}^{n-1}-Q^{*}\right\|_{2}^{2} / \kappa-C\left(\tau_{*}(t)\right)^{-\alpha} K_{\psi}^{(2)}\left\|\left(y^{n, t}, z^{n, t}\right)-\left(y^{n, *}, z^{n, *}\right)\right\|_{2} \sqrt{\left\|Q_{T}^{n-1}-Q^{*}\right\|_{2}} .
\end{aligned}
$$

Since the term $\left(1-C\left(\tau_{*}(t)\right)^{-\alpha} K_{\psi}^{(1)} \kappa\right) \|\left(y_{t}^{n}, z_{t}^{n}\right)$ decreases with $\kappa$, while the term $C\left(\tau_{*}(t)\right)^{-\alpha}\left\|Q_{T}^{n-1}-Q^{*}\right\|_{2}^{2} / \kappa$ increases with $\kappa$. We further claim that we can choose $\kappa$ satisfying $0<\kappa<1 /\left(C K_{\psi}^{(1)}\right)$ such that

$$
\left(1-C\left(\tau_{*}(t)\right)^{-\alpha} K_{\psi}^{(1)} \kappa\right)\left\|\left(y_{t}^{n}, z_{t}^{n}\right)-\left(y^{n, *}, z^{n, *}\right)\right\|_{2}^{2}-C\left(\tau_{*}(t)\right)^{-\alpha}\left\|Q_{T}^{n-1}-Q^{*}\right\|_{2}^{2} / \kappa \leq 0,
$$

which gives the desired result.

Step 2: Bounding $\left\|\left(y^{*}, z^{*}\right)-\left(y^{n, *}, z^{n, *}\right)\right\|_{2}$, for all $n \leq N$, by a function of $\left\|Q_{T}^{n-1}-Q^{*}\right\|_{2}$. We first present a well known inequality.

Fact 5.2. Given two proper functions $f_{1}, f_{2}: \mathcal{X} \rightarrow \mathbb{R}$,

$$
\left|\max _{x \in \mathcal{X}} f_{1}(x)-\max _{x \in \mathcal{X}} f_{2}(x)\right| \leq \max _{x \in \mathcal{X}}\left|f_{1}(x)-f_{2}(x)\right|
$$

and

$$
\left|\min _{x \in \mathcal{X}} f_{1}(x)-\min _{x \in \mathcal{X}} f_{2}(x)\right| \leq \max _{x \in \mathcal{X}}\left|f_{1}(x)-f_{2}(x)\right| .
$$

In particular, Fact 5.2 implies that

$$
\left|\min _{a} Q_{T}^{n-1}(s, a)-\min _{a} Q^{*}(s, a)\right| \leq\left\|Q_{T}^{n-1}-Q^{*}\right\|_{\infty} \leq\left\|Q_{T}^{n-1}-Q^{*}\right\|_{2},
$$

for all $s \in \mathbb{S}$. 
Lemma 5.3. Suppose Assumption 3.1 holds, then, for all $n \leq N$, we have

$$
\left\|\left(y^{*}, z^{*}\right)-\left(y^{n, *}, z^{n, *}\right)\right\|_{2} \leq K_{S} K_{G}\left\|Q_{T}^{n-1}-Q^{*}\right\|_{2} .
$$

Proof. It can be shown that

$$
\begin{aligned}
& \left\|\left(y^{*}, z^{*}\right)-\left(y^{n, *}, z^{n, *}\right)\right\|_{2} \\
\leq & K_{S}\left\|\mathbb{E}_{s^{\prime} \sim P(\cdot \mid s, a)}\left[G\left(v^{n-1}\left(s^{\prime}\right), y^{*}(s, a), z^{*}(s, a)\right)\right]-\mathbb{E}_{s^{\prime} \sim P(\cdot \mid s, a)}\left[G\left(v^{n-1}\left(s^{\prime}\right), y^{n, *}(s, a), z^{n, *}(s, a)\right)\right]\right\|_{2} \\
\leq & K_{S} \max _{z \in \mathcal{Z}}\left\|\min _{y \in \mathcal{Y}} \mathbb{E}_{s^{\prime} \sim P(\cdot \mid s, a)}\left[G\left(v^{n-1}\left(s^{\prime}\right), y(s, a), z(s, a)\right)\right]-\min _{y \in \mathcal{Y}} \mathbb{E}_{s^{\prime} \sim P(\cdot \mid s, a)}\left[G\left(v^{*}\left(s^{\prime}\right), y(s, a), z(s, a)\right)\right]\right\|_{2} \\
\leq & K_{S} \max _{y \in \mathcal{Y}, z \in \mathcal{Z}}\left\|\mathbb{E}_{s^{\prime} \sim P(\cdot \mid s, a)}\left[G\left(v^{n-1}\left(s^{\prime}\right), y(s, a), z(s, a)\right)\right]-\mathbb{E}_{s^{\prime} \sim P(\cdot \mid s, a)}\left[G\left(v^{*}\left(s^{\prime}\right), y(s, a), z(s, a)\right)\right]\right\|_{2} \\
\leq & K_{S} K_{G}\left\|\min _{a} Q_{t}^{n-1}(s, a)-\min _{a} Q^{*}(s, a)\right\|_{2} \\
\leq & K_{S} K_{G}\left\|Q_{t}^{n-1}-Q^{*}\right\|_{2},
\end{aligned}
$$

where the first inequality follows from Lemma 4.6 , the second inequalities holds due to Fact 5.2 the third inequality holds by Lipschitz continuity of $G$ under Assumption 3.1 (ii), fourth inequality holds based on the Lipschitz continuity of function $G$ on $\mathcal{V} \times \mathcal{Y} \times \mathcal{Z}$, and the last inequality holds by inequality (5.6).

Step 3: Apply the stochastic approximation convergence theorem. This step completes the proof of Theorem 4.4. We introduce an operator $H: \mathcal{V} \times \mathcal{Y} \times \mathcal{Z} \rightarrow \mathbb{R}^{|\mathbb{S}||\mathbb{A}|}$ defined via

$$
H(v, y, z)(s, a):=c(s, a)+\gamma G\left(v\left(s^{\prime}\right), y(s, a), z(s, a)\right), s^{\prime} \sim P(\cdot \mid s, a),
$$

for all $(s, a) \in \mathbb{K}$. Eq. 4.6 may then be written as

$$
Q^{*}(s, a)=\mathbb{E}_{s^{\prime} \sim P(\cdot \mid s, a)}\left[H\left(v^{*}, y^{*}, z^{*}\right)\right](s, a), \forall(s, a) \in \mathbb{K} .
$$

Next we define two stochastic processes,

$$
\begin{aligned}
& \epsilon_{t}^{n}(s, a):=H\left(v^{n-1}, y^{n, *}, z^{n, *}\right)(s, a)-H\left(v^{n-1}, y^{n, t}, z^{n, t}\right)(s, a), \\
& \xi_{t}^{n}(s, a):=H\left(v^{*} y^{*}, z^{*}\right)(s, a)-H\left(v^{n-1}, y^{n, *}, z^{n, *}\right)(s, a),
\end{aligned}
$$

for all $(s, a) \in \mathbb{K}, t \leq T$, and $n \leq N$, where $\epsilon_{t}^{n}$ corresponds to the risk estimation error (i.e. the duality gap of the stochastic saddle-point problem) and $\xi_{t}^{n}$ corresponds to the error between $Q_{T}^{n}$ and $Q^{*}$. Therefore, Step 3 in Algorithm 1 is equivalent to

$$
\begin{aligned}
& Q_{t}^{n}(s, a)-Q_{T}^{n-1}(s, a) \\
= & -\theta_{k}^{n}\left[Q_{T}^{n-1}(s, a)-Q^{*}(s, a)+\xi_{t}^{n}(s, a)+\epsilon_{t}^{n}(s, a)+Q^{*}(s, a)-H\left(v^{*}, y^{*}, z^{*}\right)(s, a)\right],
\end{aligned}
$$

for all $(s, a) \in \mathbb{K}, t \leq T$, and $n \leq N$. Clearly, $\mathbb{E}\left[Q^{*}(s, a)-H\left(v^{*}, y^{*}, z^{*}(s, a)\right) \mid \mathcal{G}_{t+1}^{n-1}\right]=0$ for all $(s, a) \in \mathbb{K}$ by 5.8 . By Lemma 5.1, we know that

$$
\mathbb{E}\left[\left\|\epsilon_{t}^{n}(s, a)\right\|_{2}^{2} \mid \mathcal{G}_{t+1}^{n-1}\right] \leq \frac{C K_{G}}{\kappa\left(1-C K_{\psi}^{(1)} \kappa\right)}\left\|Q_{T}^{n-1}-Q^{*}\right\|_{2}^{2}, \forall(s, a) \in \mathbb{K} .
$$

In particular, inequality (5.10) holds by setting $t=1$ in (5.1). Furthermore, inequality (5.10) shows that the conditional expectation w.r.t. $\mathcal{G}_{t+1}^{n-1}$ of the risk estimation error for each state-action pair at each iteration is always bounded by $\left\|Q_{T}^{n-1}-Q^{*}\right\|_{2}^{2}$. In addition, by Lipschitz continuity of $G$, we have

$$
\begin{aligned}
\left|\xi_{t}^{n}(s, a)\right| & \leq \gamma K_{G}\left|\min _{a \in \mathbb{A}} Q_{T}^{n-1}\left(s_{t}^{n}, a\right)-\min _{a \in \mathbb{A}} Q^{*}\left(s_{t}^{n}, a\right)\right|+\gamma K_{G}\left|\left(y^{*}(s, a), z^{*}(s, a)\right)-\left(y^{n, *}(s, a), z^{n, *}(s, a)\right)\right| \\
& \leq \gamma K_{G}\left[\left\|Q_{T}^{n-1}-Q^{*}\right\|_{2}+\left\|\left(y^{*}, z^{*}\right)-\left(y^{n, *}, z^{n, *}\right)\right\|_{2}\right] \\
& \leq \gamma K_{G}\left(1+K_{G} K_{S}\right)\left\|Q_{T}^{n-1}-Q^{*}\right\|_{2},
\end{aligned}
$$


where the final inequality holds due to Lemma 5.3 Since $G$ is $P$-square summable for every $y \in \mathcal{Y}$ and $z \in \mathcal{Z}$ in Assumption 3.1 $(\mathrm{i})$, the risk measure 4.3$)$ is bounded for all $(s, a) \in \mathbb{K}$. Furthermore, based on (4.5) and boundedness of the cost function $c(s, a)$ by Assumption 2.1(ii), we have boundedness of $Q_{t}^{n}$ for all $t \leq T, n \leq N$. Boundedness of the $Q$-values together with results (5.10) and (5.11), along with the equality $\mathbb{E}\left[Q^{*}-H\left(v^{*}, y^{*}, z^{*}\right) \mid \mathcal{G}_{t+1}^{n-1}\right]=0$, mean that the update rule 5.9 .9 satisfies the conditions of [40, Theorem 2.4] and [19, Assumption A2]. We now have the ingredients needed to apply the stochastic approximation convergence theorem (see e.g. [40, Theorem 2.4] or [19, Theorem 2.2]) to Eq. (5.9) to conclude that

$$
Q_{T}^{n}(s, a) \rightarrow Q^{*}(s, a) \quad \text { a.s. }
$$

for all $(s, a) \in \mathbb{K}$ as $n \rightarrow \infty$.

Remark 5.4. In terms of RaQL with random costs, the result of Lemma 4.5 holds for the modified subdifferentials of the function $G$ with respect to $c\left(s_{t}^{n}, a^{n}, x_{t}^{n}\right)+\gamma v^{n-1}\left(s_{t}^{n}, a^{n}\right)$ and $c\left(s_{t}^{n}, a^{n}, x_{t}^{n}\right)+\gamma v^{*}\left(s_{t}^{n}, a^{n}\right)$, based on 4.13). We can then follow Steps 1 and 2 to bound $\left\|\left(y^{n, t}, z^{n, t}\right)-\left(y^{n, *}, z^{n, *}\right)\right\|_{2}^{2}$ and $\left\|\left(y^{*}, z^{*}\right)-\left(y^{n, *}, z^{n, *}\right)\right\|_{2}$ in terms of $\left\|Q_{T}^{n-1}-Q^{*}\right\|_{2}$. Finally, we can again apply the stochastic approximation convergence theorem [40, Theorem 2.4] or [19, Theorem 2.2] to prove almost sure convergence of RaQL for random costs.

\subsection{Convergence rate}

In this subsection we derive the convergence rate of RaQL for a polynomial learning rate $k \in(1 / 2,1)$. Our convergence rate proof follows [26]. The main idea is to connect the convergence of RaQL with the convergence of an artificial deterministic sequence, which has a linear convergence rate that is easier to derive. In other words, the values $\left\{Q_{T}^{n}\right\}_{n \geq 1}$ could be bounded by a deterministic sequence almost surely in each iteration. Explicitly, we will construct $0<\beta_{T}<1$ and an artificial deterministic sequence $\left\{D_{m}\right\}_{m \geq 1}$ satisfying $D_{0}=D_{1}=V_{\max }$ and $D_{m+1}=\left(1-\beta_{T}\right) D_{m}$ for all $m \geq 1$. Here we call $m$ as "epoch". Clearly, the sequence $\left\{D_{m}\right\}_{m \geq 1}$ converges to zero. This sequence also has the following special property: for every $m \geq 1$, there exists $\tau_{m}$ such that $\left\|Q_{T}^{n}-Q^{*}\right\|_{2} \leq D_{m}$ holds for all $n \geq \tau_{m}$. The duration of epoch $m$ is then $\tau_{m+1}-\tau_{m}$. Subsequently, we show that $Q_{t}^{n}(s, a)-Q^{*}(s, a)$ is bounded by two simpler stochastic processes $\left\{Z_{t}^{n, \tau_{m}}(s, a)\right\}_{\tau_{m} \leq n \leq N}$ and $\left\{Y_{t}^{n, \tau_{m}}(s, a)\right\}_{\tau_{m} \leq n \leq N}$. We then establish the relationship of $\left\{Z_{t}^{n, \tau_{m}}(s, a)\right\}_{\tau_{m} \leq n \leq N}$ and $\left\{Y_{t}^{n, \tau_{m}}(s, a)\right\}_{\tau_{m} \leq n \leq N}$ with $\left\{D_{m}\right\}_{m \geq 1}$. In particular, $\left\{Z_{t}^{l, \tau_{m}}(s, a)\right\}_{l=1, \ldots, n}$ is a martingale difference sequence so we can derive a high probability bound on $\left\{Z_{t}^{n, \tau_{m}}(s, a)\right\}_{\tau_{m} \leq n \leq N}$ from the Azuma-Hoeffding inequality. On the other hand, $\left\{Y_{t}^{n, \tau_{m}}(s, a)\right\}_{\tau_{m} \leq n \leq N}$ captures all of the biased estimation error terms (from the risk estimation error and the $Q$-value estimation error) in RaQL, which can be bounded almost surely by a function of $\left\{D_{m}\right\}_{m \geq 1}$. By combining these two results, we show that $\left\|Q_{T}^{N}-Q^{*}\right\|_{2} \leq \tilde{\varepsilon}$ holds with high probability for large enough $N$ and any $T \geq 1$.

We will verify the existence and provide the explicit forms of: $D_{m}, \beta_{T}$, and $\tau_{m}$ in the upcoming steps.

Step 1: Constructing two stochastic processes and bounding $\left\|Q_{t}^{n}-Q^{*}\right\|_{2}$ by their sum. We decompose 5.9 into two separate stochastic processes $\left\{Z_{t}^{n, \tau_{m}}(s, a)\right\}_{\tau_{m} \leq n \leq N}$ and $\left\{Y_{t}^{n, \tau_{m}}(s, a)\right\}_{\tau_{m} \leq n \leq N}$. We define, for a fixed $m \geq 1$ and for $n \geq \tau_{m}$ and $t \leq T$, the quantity

$$
Z_{t}^{n+1, \tau_{m}}(s, a):=\left(1-\theta_{k}^{n}(s, a)\right) Z_{t}^{n, \tau_{m}}(s, a)+\theta_{k}^{n}(s, a) \zeta(s, a),
$$

for all $(s, a) \in \mathbb{K}$, where $\zeta(s, a):=Q^{*}(s, a)-H\left(v^{*}, y^{*}, z^{*}\right)(s, a)$ and $Z_{t}^{\tau_{m}, \tau_{m}}=0$, for all $t \leq T$. We also define, for a fixed $m \geq 1$ and for $n \geq \tau_{m}$ and $t \leq T$, the quantity

$$
\begin{aligned}
Y_{t}^{n+1, \tau_{m}}(s, a):= & \left(1-\theta_{k}^{n}(s, a)\right) Y_{t}^{n, \tau_{m}}(s, a)+\theta_{k}^{n}(s, a) K_{G} \gamma\left(D_{m}+\left\|\left(y^{*}, z^{*}\right)-\left(y^{n, *}, z^{n, *}\right)\right\|_{2}\right) \\
& +\theta_{k}^{n}(s, a) K_{G}\left\|\left(y^{n, t}, z^{n, t}\right)-\left(y^{n, *}, z^{n, *}\right)\right\|_{2},
\end{aligned}
$$

for all $(s, a) \in \mathbb{K}$, where $Y_{t}^{\tau_{m}, \tau_{m}}(s, a)=D_{m}$, for all $t \leq T$. The process 5.12 is a recursion for the unbiased error terms $\zeta(s, a)$, while the process 5.13 is a recursion for the biased error terms (e.g. the sum of the $Q$-value approximation errors and the duality gaps from the risk estimation). The following Lemma 5.5 which appears in [14] and 26, Lemma 9] shows that the almost sure lower and upper bounds for $Q$-value estimation error at each iteration by the process 5.13 and 5.12 . 
Lemma 5.5. Given the stochastic processes (5.12) and (5.13), and the update rule (5.9),

$$
Z_{t}^{n, \tau_{m}}(s, a)-Y_{t}^{n, \tau_{m}}(s, a) \leq Q_{t}^{n}(s, a)-Q^{*}(s, a) \leq Z_{t}^{n, \tau_{m}}(s, a)+Y_{t}^{n, \tau_{m}}(s, a),
$$

holds for all $t \leq T, \tau_{m} \leq n \leq N$, and $(s, a) \in \mathbb{K}$.

Proof. Suppose both $\epsilon_{t}^{n}(s, a)$ and $\xi_{t}^{n}(s, a)$ are non-negative for all $(s, a) \in \mathbb{K}$. From the right hand side of Eq. (5.13), for all $t \leq T, n \leq N$, we have

$$
\begin{aligned}
& \left(1-\theta_{k}^{n}(s, a)\right) Y_{t}^{n, \tau_{m}}(s, a)+\theta_{k}^{n}(s, a) K_{G} \gamma\left(D_{m}+\left\|\left(y^{*}, z^{*}\right)-\left(y^{n, *}, z^{n, *}\right)\right\|_{2}\right) \\
& +\theta_{k}^{n}(s, a) K_{G}\left\|\left(y^{n, t}, z^{n, t}\right)-\left(y^{n, *}, z^{n, *}\right)\right\|_{2} \\
\geq & \left(1-\theta_{k}^{n}(s, a)\right) Y_{t}^{n, \tau_{m}}(s, a)+\theta_{k}^{n}(s, a)\left(\left|\xi_{t}^{n}(s, a)\right|+\left|\epsilon_{t}^{n}(s, a)\right|\right) \\
= & \left(1-\theta_{k}^{n}(s, a)\right) Y_{t}^{n, \tau_{m}}(s, a)+\theta_{k}^{n}(s, a)\left(\xi_{t}^{n}(s, a)+\epsilon_{t}^{n}(s, a)\right),
\end{aligned}
$$

where this inequality is due to inequality (5.11) $\left(\left|\xi_{t}^{n}(s, a)\right| \leq \gamma K_{G}\left[\left\|Q_{T}^{n-1}-Q^{*}\right\|_{2}+\left\|\left(y^{*}, z^{*}\right)-\left(y^{n, *}, z^{n, *}\right)\right\|_{2}\right]\right)$, the definition of $\epsilon_{t}^{n}(s, a)$, and Lipschitz continuity of $G$ from Assumption 3.1 (ii) $\left(\left|\epsilon_{t}^{n}(s, a)\right| \leq K_{G} \|\left(y^{n, t}, z^{n, t}\right)-\right.$ $\left.\left(y^{n, *}, z^{n, *}\right) \|_{2}\right)$. Combining inequality (5.15) with Eqs. (5.13) and (5.12), for all $t \leq T, n \leq N$, and $(s, a) \in \mathbb{K}$, we have

$$
\begin{aligned}
& Z_{t}^{n+1, \tau_{m}}(s, a)+Y_{t}^{n+1, \tau_{m}}(s, a) \\
\geq & \left(1-\theta_{k}^{n}(s, a)\right)\left(Z_{t}^{n, \tau_{m}}(s, a)+Y_{t}^{n, \tau_{m}}(s, a)\right) \\
& +\theta_{k}^{n}(s, a)\left[\xi_{t}^{n}(s, a)+\epsilon_{t}^{n}(s, a)+Q^{*}(s, a)-H\left(v^{*}, y^{*}, z^{*}\right)(s, a)\right] .
\end{aligned}
$$

We now use induction on $n$ to show that the right-hand side in inequalities (5.14) holds. By setting the base case to be $n=\tau_{m}$, we have, for all $t \leq T$ and $(s, a) \in \mathbb{K}$, that

$$
Z_{t}^{\tau_{m}, \tau_{m}}(s, a)+Y_{t}^{\tau_{m}, \tau_{m}}(s, a)=D_{m} \geq\left\|Q_{t}^{\tau_{m}}-Q^{*}\right\|_{2} \geq Q_{t}^{n}(s, a)-Q^{*}(s, a) .
$$

The above equality holds by the definition of $Z_{t}^{n}(s, a)$ and $Y_{t}^{n}(s, a)$ in Eqs. (5.12) and (5.13), respectively. Suppose $Z_{t}^{n, \tau_{m}}(s, a)+Y_{t}^{n, \tau_{m}}(s, a) \geq Q_{t}^{n}(s, a)-Q^{*}(s, a)$ for all $\tau_{m} \leq n \leq N, t \leq T$, and $(s, a) \in \mathbb{K}$. Then, by inequality (5.16) and Eq. (5.9), we obtain the right hand side of the above inequality. Now, suppose both $\epsilon_{t}^{n}(s, a)$ and $\xi_{t}^{n}(s, a)$ are negative for all $(s, a) \in \mathbb{K}$, then

$$
\begin{aligned}
& -\left(1-\theta_{k}^{n}(s, a)\right) Y_{t}^{n, \tau_{m}}(s, a)+\theta_{k}^{n}(s, a) K_{G} \gamma\left(-D_{m}-\left\|\left(y^{*}, z^{*}\right)-\left(y^{n, *}, z^{n, *}\right)\right\|_{2}\right) \\
& -\theta_{k}^{n}(s, a) K_{G}\left\|\left(y^{n, t}, z^{n, t}\right)-\left(y^{n, *}, z^{n, *}\right)\right\|_{2} \\
\leq & -\left(1-\theta_{k}^{n}(s, a)\right) Y_{t}^{n, \tau_{m}}(s, a)+\theta_{k}^{n}(s, a)\left(\xi_{t}^{n}(s, a)+\epsilon_{t}^{n}(s, a)\right),
\end{aligned}
$$

and so we obtain the left hand inequality in inequalities (5.14) following the same reasoning. Finally, when $\epsilon_{t}^{n}(s, a)$ and $\xi_{t}^{n}(s, a)$ have different signs for all $(s, a) \in \mathbb{K}$, we can show that inequalities (5.15) and (5.17) hold. Thus, following the same inductive reasoning, we can show that both inequalities in (5.14) hold.

Our main focus is on deriving a high probability bound for the convergence rate of $\left\|Q_{T}^{n}-Q^{*}\right\|_{2}$. By Lemma (5.5), this goal is equivalent to bounding the sum and the difference of the stochastic processes $\left\{Z_{t}^{n, \tau_{m}}(s, a)\right\}_{\tau_{m} \leq n \leq N}$ and $\left\{Y_{t}^{n, \tau_{m}}(s, a)\right\}_{\tau_{m} \leq n \leq N}$. In the following steps of the proof, we first derive almost sure bounds on $\left\{Y_{T}^{n, \tau_{m}}\right\}_{\tau_{m} \leq n \leq N}$.

Step 2: Bounding $\left\{Y_{T}^{n, \tau_{m}}\right\}_{\tau_{m} \leq n \leq N}$ and selecting $\beta_{T}$. The next lemma provides an almost sure upper bound on the stochastic process $\left\{Y_{T}^{n, \tau_{m}}\right\}_{\tau_{m} \leq n \leq N}$. Furthermore, this lemma shows that the duration of epoch $m$, which starts at time $\tau_{m}$ and ends at time $\tau_{m+1}$, is bounded by $\left(\tau_{m}\right)^{k}$, and it also provides an explicit selection of $\beta_{T}$.

Lemma 5.6. Given any $m \geq 1$, assume that for all $n \geq \tau_{m}$, we have $Y_{T}^{n, \tau_{m}}(s, a) \leq D_{m}$. Then, for any $n \geq \tau_{m}+\left(\tau_{m}\right)^{k}=\tau_{m+1}$ we have

$$
Y_{T}^{n, \tau_{m}}(s, a) \leq K_{G} D_{m}\left(\gamma+\sqrt{\frac{C\left(\tau_{*}(T)\right)^{-\alpha}}{\kappa\left(1-C\left(\tau_{*}(T)\right)^{-\alpha} K_{\psi}^{(1)} \kappa\right)}}+K_{G} K_{S}\right)+\frac{2}{e} \beta_{T} D_{m},
$$

for all $(s, a) \in \mathbb{K}$, where $\beta_{T}$ is given in Eq. (4.15) with $T$ satisfies conditions (4.16) and (4.17). 
Proof. Based on the proof in [26. Lemma 27], we combine the convergence rate results from Lemmas 5.1 and 5.3 into the definition of the process $Y_{T}^{n+1, \tau_{m}}$ as described in Eq. 5.13. We then obtain

$$
Y_{T}^{\tau_{m}, \tau_{m}}(s, a)=K_{G} D_{m}\left(\gamma+\sqrt{\frac{C\left(\tau_{*}(T)\right)^{-\alpha}}{\kappa\left(1-C\left(\tau_{*}(T)\right)^{-\alpha} K_{\psi}^{(1)} \kappa\right)}}+K_{G} K_{S}\right)+g_{\tau_{m}},
$$

for all $(s, a) \in \mathbb{K}$ and $m \geq 1$, where

$$
g_{\tau_{m}}:=K_{G}\left\{1-\gamma-\sqrt{\frac{C\left(\tau_{*}(T)\right)^{-\alpha}}{\kappa\left(1-C\left(\tau_{*}(T)\right)^{-\alpha} K_{\psi}^{(1)} \kappa\right)}}-K_{G} K_{S}\right\} D_{m},
$$

for all $(s, a) \in \mathbb{K}$ and $m \geq 1$. We may then write

$$
Y_{T}^{n, \tau_{m}}(s, a)=K_{G} D_{m}\left(1-\gamma-\sqrt{\frac{C\left(\tau_{*}(T)\right)^{-\alpha}}{\kappa\left(1-C\left(\tau_{*}(T)\right)^{-\alpha} K_{\psi}^{(1)} \kappa\right)}}-K_{G} K_{S}\right)+\left(1-\theta_{k}^{n}\right) g_{n},
$$

for all $(s, a) \in \mathbb{K}, n \leq N$ and $m \geq 1$. Since the step sizes $\theta_{k}^{n}$ are monotonically decreasing in $n$, we can rewrite $g_{n}$ as

$$
\begin{aligned}
g_{n} & =K_{G}\left\{1-\gamma-\sqrt{\frac{C\left(\tau_{*}(T)\right)^{-\alpha}}{\kappa\left(1-C\left(\tau_{*}(T)\right)^{-\alpha} K_{\psi}^{(1)} \kappa\right)}}-K_{G} K_{S}\right\} D_{m} \prod_{l=1}^{n-\tau_{m}}\left(1-\theta_{k}^{l+\tau_{m}}\right) \\
& \leq 2 \beta_{T} D_{m} \prod_{l=1}^{n-\tau_{m}}\left(1-\frac{1}{\left(l+\tau_{m}\right)^{k}}\right) \\
& \leq 2 \beta_{T} D_{m} \prod_{l=1}^{n-\tau_{m}}\left(1-\left(\frac{1}{\tau_{m}}\right)^{k}\right)^{n-\tau_{m}} \\
& \leq 2 \beta_{T} D_{m}\left(1-\left(\frac{1}{\tau_{m}}\right)^{k}\right)^{\left(\tau_{m}\right)^{k}} \\
& \leq \frac{2}{e} \beta_{T} D_{m}
\end{aligned}
$$

for all $n \leq N$, where the first inequality holds by the choice of $\beta_{T}$ in Eq. 4.15.

Step 3: Deriving high probability bound on $\left\{\left|Z_{T}^{n, \tau_{m}}\right|\right\}_{\tau_{m} \leq n \leq N}$ by the Azuma-Hoeffding inequality. The following Lemma 5.7 directly follows from the results in [26, Lemma 28]. It shows that $\left\{Z_{t}^{l, \tau_{m}}(s, a)\right\}_{l=1, \ldots, n}$ for fixed $n \leq N$, is a martingale difference sequence for all $(s, a) \in \mathbb{K}$.

Lemma 5.7. [26, Lemma 28] Given a fixed $n \geq \tau_{m}$, for any $i \in\left[\tau_{m}, n\right]$, define

$$
\eta_{i}^{m, n}(s, a):=\theta_{k}^{i+\tau_{m}}(s, a) \prod_{j=i+\tau_{m}+1}^{n}\left[1-\zeta_{t+1}^{j}(s, a)\right] .
$$

Let $\tilde{w}_{i+\tau_{m}}^{n}(s, a):=\eta_{i}^{m, n}(s, a) \zeta_{t+1}^{i+\tau_{m}}(s, a)$ so that $\left|Z_{t}^{l, \tau_{m}}(s, a)\right|=\sum_{i=1}^{l} \tilde{w}_{i+\tau_{m}}^{n}(s, a)$. Then, for all $(s, a) \in \mathbb{K}$, we have: (i) for any $n \in\left[\tau_{m+1}, \tau_{m+2}\right]$, the random variable $\tilde{w}_{i+\tau_{m}}^{n}(s, a)$ has zero mean and is bounded by $\left((1-\varepsilon)^{k}\left(\tau_{m}\right)^{k}\right)^{-1} V_{\max }$; (ii) for any $n \in\left[\tau_{m+1}, \tau_{m+2}\right]$ and $1 \leq l \leq n, Z_{t}^{l, \tau_{m}}(s, a)$ is a martingale difference sequence satisfying $\left|Z_{t}^{l, \tau_{m}}(s, a)-Z_{t}^{l-1, \tau_{m}}(s, a)\right| \leq\left((1-\varepsilon)^{k}\left(\tau_{m}\right)^{k}\right)^{-1} V_{\max }$.

Based on Lemma 5.7 and [6], we obtain a high probability bound on $\left|Z_{T}^{n, \tau_{m}}(s, a)\right|$ w.r.t. $m \geq 1$ and $n \in\left[\tau_{m+1}, \tau_{m+2}\right]$ by the Azuma-Hoeffding inequality, we also derive a selection rule for choosing $\tau_{0}$ and $\left\{\tau_{m}\right\}_{m \geq 1}$.

Lemma 5.8. Given $0<\delta<1$, we have: (i)

$$
\mathbb{P}\left[\forall n \in\left[\tau_{m+1}, \tau_{m+2}\right]: \forall(s, a) \in \mathbb{K}:\left|Z_{T}^{n, \tau_{m}}(s, a)\right| \leq\left(1-\frac{2}{e}\right) \beta_{T} D_{m}\right] \geq 1-\delta(1-\varepsilon),
$$


for

$$
\tau_{m}=\Theta\left(\left(\frac{V_{\max }^{2} \ln \left(V_{\max }|\mathbb{S} \| \mathbb{A}| /\left[\delta \beta_{T} D_{m}(1-\varepsilon)\right]\right)}{\left(\beta_{T}\right)^{2} D_{m}(1-\varepsilon)^{1+3 k}}\right)^{1 / k}\right),
$$

and (ii)

$$
\mathbb{P}\left[\forall m \in\left[1, \frac{1}{1-\varepsilon}\right], \forall n \in\left[\tau_{m+1}, \tau_{m+2}\right], \forall(s, a) \in \mathbb{K}:\left|Z_{T}^{n, \tau_{m}}(s, a)\right| \leq \tilde{\varepsilon}\right] \geq 1-\delta,
$$

for

$$
\tau_{0}=\Theta\left(\left(\frac{V_{\max }^{2} \ln \left(V_{\max }|\mathbb{S}||\mathbb{A}| /\left[\delta \beta_{T} \tilde{\varepsilon}(1-\varepsilon)\right]\right)}{\left(\beta_{T}\right)^{2} \tilde{\varepsilon}^{2}(1-\varepsilon)^{1+3 k}}\right)^{1 / k}\right) .
$$

Proof. First, note that $\left\{Z_{t}^{l, \tau_{m}}(s, a)\right\}_{l=1, \ldots, n}$ is a martingale difference sequence for all $(s, a) \in \mathbb{K}$ by Lemma 5.7. Next, following the proofs of [26. Lemma 30] and [26, Lemma 31], for each state-action pair, we apply Lemma 5.7 and the Azuma-Hoeffding inequality to $Z_{T}^{n, \tau_{m}}(s, a)$ with $c_{i}=\frac{1}{(1-\varepsilon)^{k}\left(\tau_{m}\right)^{k}} V_{\max }$, for all $i \in\left[\tau_{m}, n\right]$. Then, for any $n \in\left[\tau_{m+1}, \tau_{m+2}\right]$, we have

$$
\begin{aligned}
\mathbb{P}\left[\left|Z_{T}^{n, \tau_{m}}(s, a)\right| \geq \tilde{\varepsilon} \mid n \in\left[\tau_{m+1}, \tau_{m+2}\right]\right] & \leq 2 \exp \left(\frac{-\tilde{\varepsilon}^{2}}{2 \sum_{i=\tau_{m}+1, i \in N^{s, a}}^{n} c_{i}^{2}}\right) \\
& \leq 2 \exp \left(-C \frac{\tilde{\varepsilon}^{2} \tau_{m}(1-\varepsilon)^{1+3 k}}{V_{\max }^{2}}\right),
\end{aligned}
$$

with a constant $C>0$. Let $\tilde{\delta}_{m}$ denote the right hand side of the inequality 5.19 , which holds for $\tau_{m}=$ $\Theta\left(\ln \left(1 / \tilde{\delta}_{m}\right) V_{\max }^{2} /(1-\varepsilon)^{1+3 k} \tilde{\varepsilon}^{2}\right)$. The union bound gives

$$
\mathbb{P}\left[\forall n \in\left[\tau_{m+1}, \tau_{m+2}\right]: Z_{T}^{n, \tau_{m}}(s, a) \leq \tilde{\varepsilon}\right] \leq \sum_{n=\tau_{m}+1}^{\tau_{m}+2} \mathbb{P}\left[Z_{T}^{n, \tau_{m}}(s, a) \leq \tilde{\varepsilon}\right],
$$

and so taking $\tilde{\delta}_{m}=\frac{\delta(1-\varepsilon)}{\left(\tau_{m+2}-\tau_{m+1}\right)|\mathbb{S}||A|}$ assures that with probability at least $1-\delta(1-\varepsilon)$, we have $\left|Z_{T}^{n, \tau_{m}}(s, a)\right| \leq$ $\tilde{\varepsilon}$ for all $(s, a) \in \mathbb{K}$ and $n \in\left[\tau_{m+1}, \tau_{m+2}\right]$. As a result, we have

$$
\tau_{m}=\Theta\left(\ln \left(1 / \tilde{\delta}_{m}\right) V_{\max }^{2} /(1-\varepsilon)^{1+3 k} \tilde{\varepsilon}^{2}\right)=\Theta\left(\left(\frac{V_{\max }^{2} \ln \left(V_{\max }|\mathbb{S}||\mathbb{A}| /\left[\delta \beta_{T} D_{m}(1-\varepsilon)\right]\right)}{\left(\beta_{T}\right)^{2} D_{m}(1-\varepsilon)^{1+3 k}}\right)^{1 / k}\right) .
$$

Setting $\tilde{\varepsilon}=(1-2 / e) \beta_{T} D_{m}$ gives the desired bound in Lemma 5.8(i). For Lemma 5.8(ii), we know that

$$
\mathbb{P}\left[\forall n \in\left[\tau_{m+1}, \tau_{m+2}\right]:\left|Z_{T}^{n, \tau_{m}}\right| \geq\left(1-\frac{2}{e}\right) \beta_{T} D_{m}\right] \leq \frac{\delta}{m},
$$

and obviously

$$
\mathbb{P}\left[\forall n \in\left[\tau_{m+1}, \tau_{m+2}\right]:\left|Z_{T}^{n, \tau_{m}}\right| \geq D_{m}\right] \leq \frac{\delta}{m} .
$$

Using the union bound again shows that

$$
\mathbb{P}\left[\forall m \leq \frac{1}{1-\varepsilon}, \forall n \in\left[\tau_{m+1}, \tau_{m+2}\right],\left|Z_{T}^{n, \tau_{m}}\right| \geq \tilde{\varepsilon}\right] \leq \sum_{m=1}^{M} \mathbb{P}\left[\forall n \in\left[\tau_{m+1}, \tau_{m+2}\right],\left|Z_{T}^{n, \tau_{m}}\right| \geq \tilde{\varepsilon}\right] \leq \delta,
$$

where $\tilde{\varepsilon}=D_{m}$. We replace $D_{m}$ with $\tilde{\varepsilon}$ in Lemma 5.8 (i) to obtain

$$
\tau_{0}=\Theta\left(\left(\frac{V_{\max }^{2} \ln \left(V_{\max }|\mathbb{S}||\mathbb{A}| /\left[\delta \beta_{T} \tilde{\varepsilon}(1-\varepsilon)\right]\right)}{\left(\beta_{T}\right)^{2} \tilde{\varepsilon}^{2}(1-\varepsilon)^{1+3 k}}\right)^{1 / k} \cdot\right)
$$


Step 4: Completing the proof by combining Steps 1 through 3. This step completes the proof of Theorem 4.7. The following Lemma 5.9 is a standard fact about numerical sequences that is used to derive the final convergence rate in Theorem 4.7

Lemma 5.9. [26, Lemma 32] Let $a_{m+1}=a_{m}+\frac{1}{1-\varepsilon}\left(a_{m}\right)^{k}=a_{0}+\sum_{i=0}^{m} \frac{1}{1-\varepsilon}\left(a_{i}\right)^{k}$. Then, for any $k \in(0,1)$, $a_{m}=O\left(\left(\left(a_{0}\right)^{k}+\frac{1}{1-\varepsilon} m\right)^{\frac{1}{1-k}}\right)=O\left(a_{0}+\left(\frac{1}{1-\varepsilon} m\right)^{\frac{1}{1-k}}\right)$.

Based on Lemma 5.9, we set $a_{0}$ to be $\tau_{0}$ in Lemma 5.8, and we have

$$
\begin{aligned}
& \mathbb{P}\left[\forall n \in\left[\tau_{m+1}, \tau_{m+2}\right]: \forall(s, a) \in \mathbb{K}:\left|Z_{T}^{n, \tau_{m}}(s, a)\right| \leq\left(1-\frac{2}{e}\right) \beta_{T} D_{m}\right] \\
\leq & \mathbb{P}\left[\forall n \in\left[\tau_{m+1}, \tau_{m+2}\right]: \forall(s, a) \in \mathbb{K}:\left|Z_{T}^{n, \tau_{m}}(s, a)\right|+Y_{T}^{n, \tau_{m}}(s, a) \leq D_{m+1}\right] \\
\leq & \mathbb{P}\left[\forall n \in\left[\tau_{m+1}, \tau_{m+2}\right]:\left\|Q_{T}^{n}-Q^{*}\right\|_{\infty} \leq D_{m+1}\right] \\
\leq & \mathbb{P}\left[\forall n \in\left[\tau_{m+1}, \tau_{m+2}\right]:\left\|Q_{T}^{n}-Q^{*}\right\|_{\infty} \leq D_{m}\right] \\
\leq & \mathbb{P}\left[\forall n \in\left[\tau_{m+1}, \tau_{m+2}\right]:\left\|Q_{T}^{n}-Q^{*}\right\|_{2} \leq \sqrt{|\mathbb{S}||\mathbb{A}|} D_{m}\right],
\end{aligned}
$$

where the first above inequality holds based on Lemma 5.6, the second one holds based on Lemma 5.5 and the third one holds based on the definition of sequence $\left\{D_{m}\right\}_{m \geq 1}$. Choose $\bar{\varepsilon}$ to satisfy $\left(1-\frac{2}{e}\right) \beta_{T} D_{m}=\bar{\varepsilon} \leq D_{m}$, then we have by Lemma 5.8(ii) that

$$
\mathbb{P}\left[\forall m \in\left[1, \frac{1}{1-\varepsilon}\right], \forall n \in\left[\tau_{m+1}, \tau_{m+2}\right], \forall(s, a) \in \mathbb{K}:\left\|Q_{T}^{n}-Q^{*}\right\|_{2} \leq \sqrt{|\mathbb{S}||\mathbb{A}|} D_{m}\right] \geq 1-\delta,
$$

with

$$
\tau_{0}=\Theta\left(\left(\frac{V_{\max }^{2} \ln \left(V_{\max }|\mathbb{S}||\mathbb{A}| /\left[\delta \beta_{T} \bar{\varepsilon}(1-\varepsilon)\right]\right)}{\left(\beta_{T}\right)^{2}(\bar{\varepsilon})^{2}(1-\varepsilon)^{1+3 k}}\right)^{1 / k}\right) .
$$

Since this statement holds for all $m \in\left[1, \frac{1}{1-\varepsilon}\right]$, based on Lemma 5.9 , we have

$$
\mathbb{P}\left[\forall n \in\left[\tau_{m+1}, \tau_{m+2}\right], \forall(s, a) \in \mathbb{K}:\left\|Q_{T}^{n}-Q^{*}\right\|_{2} \leq \sqrt{|\mathbb{S}||\mathbb{A}|} D_{m}\right] \geq 1-\delta .
$$

Set $\tilde{\varepsilon}$ such that $\sqrt{|\mathbb{S}||\mathbb{A}|} D_{m} \leq \tilde{\varepsilon}$ and $D_{m}=V_{\max }\left(1-\beta_{T}\right)^{m}$, we have $m \geq\left(1 / \beta_{T}\right) \ln \left(V_{\max } \sqrt{|\mathbb{S}||\mathbb{A}|} / \tilde{\varepsilon}\right)$ and so

$$
\tau_{m}=\Theta\left(\left(\frac{V_{\max }^{2} \ln \left(V_{\max }|\mathbb{S}||\mathbb{A}| /\left[\delta \beta_{T} \bar{\varepsilon}(1-\varepsilon)\right]\right)}{\left(\beta_{T}\right)^{2}(\bar{\varepsilon})^{2}(1-\varepsilon)^{1+3 k}}\right)^{1 / k}+\left(\frac{1}{(1-\varepsilon) \beta_{T}} \ln \left(\frac{V_{\max } \sqrt{|\mathbb{S}||\mathbb{A}|}}{\tilde{\varepsilon}}\right)\right)^{\frac{1}{1-k}}\right) .
$$

Since the probability bound $\left[5.20\right.$ holds for all $n \in\left[\tau_{m+1}, \tau_{m+2}\right]$, if we replace $\bar{\varepsilon}$ with $\tilde{\varepsilon} / \sqrt{|\mathbb{S}||\mathbb{A}|}$, we get the desired result since $\bar{\varepsilon} \leq \tilde{\varepsilon} / \sqrt{|\mathbb{S}||\mathbb{A}|}$.

\section{Numerical experiments}

We illustrate the application of RaQL with an infinite-horizon inventory control problem. In practice, RaQL finds the optimal risk-aware ordering policy $\pi^{*}: \mathbb{S} \rightarrow \mathbb{A}$, which is more reliable than the standard one because it is sensitive to low probability events with extremely high random demand. In each stage, we first observe the current stock $s \in \mathbb{S}$ in inventory, then order $a \in \mathbb{A}$ new units, after which a random demand $D$ is realized. The new inventory level in the next stage is

$$
s^{\prime}=\max \left\{0, \min \left\{s+a, S_{\max }\right\}-D\right\},
$$

where $S_{\max }$ denotes the largest state in $\mathbb{S}:=\left\{0,1, \ldots, S_{\max }\right\}$. The random cost is

$$
c(s, a, D)=\tilde{c} \cdot a+b \cdot \max \{D-s-a, 0\}-p \cdot \min \{s+a, D\},
$$


where $\tilde{c}, p$ and $b$ are the unit order cost, selling price, and backorder cost, respectively.

For our experiments, we choose $\tilde{c}=3, p=5, b=4, D$ is uniform on $\{1,2, \ldots, 10\}$, and the finite state/action spaces are: $\mathbb{S}=\{0,1, \ldots, 19\}$ and $\mathbb{A}=\{1,2, \ldots, 10\}$. We set the discount factor to be $\gamma=0.1$, and we assume that all model parameters (costs, price, and transition probabilities) are all stationary. In these experiments, we evaluate the performance in terms of the relative error $\left\|Q_{T}^{n}-Q^{*}\right\|_{2} /\left\|Q^{*}\right\|_{2}, n \leq N$. Here, we obtain $Q^{*}$ exactly by doing risk-aware DP (as proposed in [57]) where in each iteration the riskaware Bellman operator is computed by exactly solving a stochastic saddle-point optimization problem (see $\mathcal{T}_{G}$ in (4.4)). First, we verify the convergence of our algorithm for a few different risk measures, and then we compare the performance with standard risk-neutral $Q$-learning. These results confirm the almost sure convergence of our algorithm as well as its competitive convergence rate. We record and compare the computation time required to reach the same relative error for RaQL with CVaR and standard $Q$-learning. We also compare the reliability of risk-aware policy and risk-neutral policy by showing how the risk-aware policy reduces the variance of expected cost when the demand is generated from the underlying distribution. Second, we test the performance of our algorithm against risk-sensitive $Q$-learning (RsQL) as proposed in [66] for the entropic risk measure, since both methods can be applied. This comparison reveals the advantages of RaQL both in terms of computational efficiency and accuracy. Third, we compare SASP and stochastic subgradient descent for risk estimation. This comparison demonstrates that SASP is better suited for estimation of complex risk measures.

Throughout the experiments, we conduct 50 simulation runs for each implementation of $Q$-learning type algorithms (RaQL, standard $Q$-learning, and RsQL), and record the mean and standard deviation of relative errors among the simulation runs. The experiments were performed on a generic laptop with Intel Core i7 processor, 8GM RAM, on a 64-bit Windows 8 operating system running Matlab R2015a and CPLEX Studio 12.5 .

\subsection{Experiment I: Risk-aware vs. Risk-neutral}

\subsubsection{Convergence rate comparison}

We intend to show that a variety of risk measures fit into our RaQL framework, and also to show that RaQL has a convergence rate similar to risk-neutral $Q$-learning. We consider CVaR and absolute semi-deviation. We set the number of outer iterations to be $N=10000$, and the number of inner iterations to be $T=100$. In these experiments, Risk-aware DP terminates after finding an $\epsilon$-optimal policy with $\epsilon=0.01$. We use a linear learning rate i.e. $k=1$, and set $\alpha=0.1$ for CVaR, and $r=0.5$ for absolute semi-deviation.

As shown in Figure 1, RaQL converges almost surely to the optimal $Q$-value as expected. Moreover, in this experiment, the convergence rate of RaQL matches classical $Q$-learning. In Figure 1, the error bars represent the standard deviation from simulation.

\subsubsection{Computation time comparison}

In this experiment, we compare the time required for RaQL and standard $Q$-learning to reach the same precision $\epsilon$ i.e. $\left\|Q_{T}^{n}-Q^{*}\right\|_{2} /\left\|Q^{*}\right\|_{2} \leq \epsilon$. Table 2 shows the expected computation time results under 50 simulations when choosing different precision levels $\epsilon$, and different $T$. Table 2 shows that for any $\epsilon$, the expected computation time for RaQL will decrease with the $T$ selected, and will be close to that of standard $Q$-learning, which means that RaQL has robust convergence even when the number of iterations for risk estimation is small.

\subsubsection{Policy comparison}

Figure 2 compares the risk-aware ordering policy from RaQL (with CVaR) and the risk-neutral ordering policy from standard $Q$-learning over 500 simulated trajectories. The histograms in Plot 4 show that the risk-aware ordering policy leads to slightly higher expected cost but lower variance. In addition, the right tails of these two distributions reveal that the risk-aware ordering policy is more reliable since it reduces the probability of events with extremely high cost. 


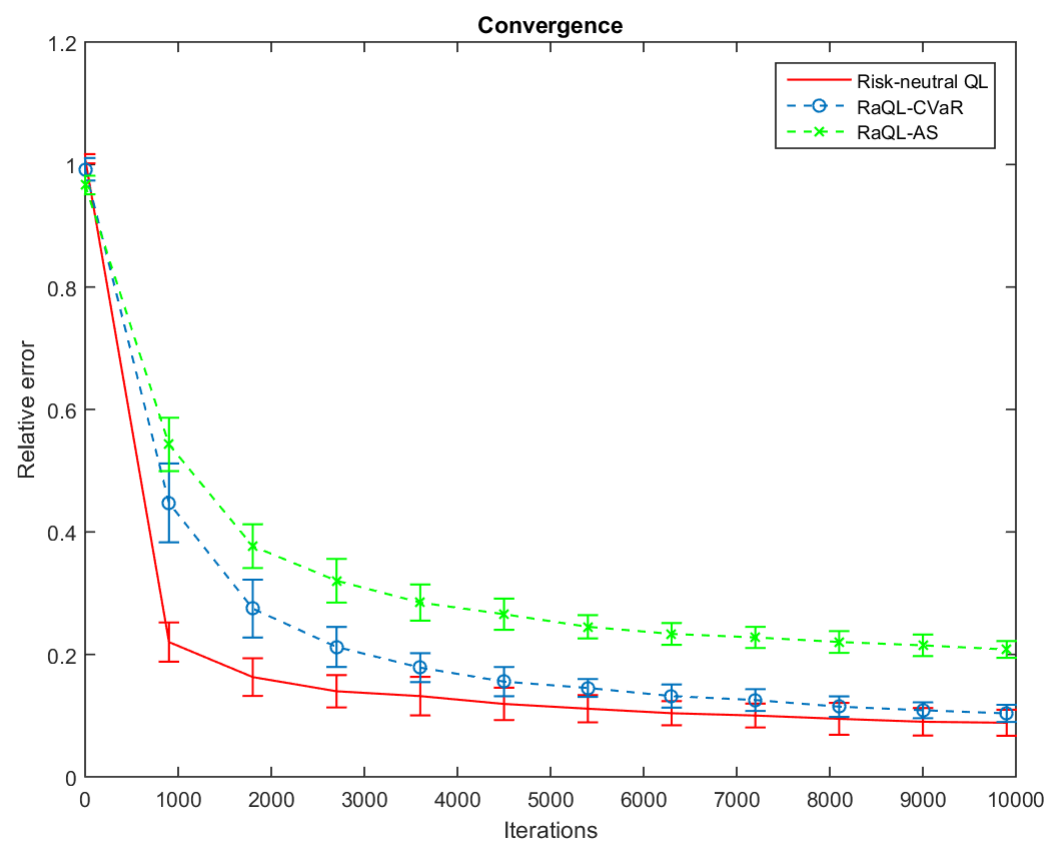

Figure 1: Numerical Experiment Result I

\begin{tabular}{ccccc}
\cline { 2 - 5 } & $\epsilon=0.5$ & $\epsilon=0.2$ & $\epsilon=0.15$ & $\epsilon=0.1$ \\
\hline $\operatorname{RaQL}(T=100)$ & $1.268 \mathrm{~s}$ & $3.493 \mathrm{~s}$ & $6.798 \mathrm{~s}$ & $30.976 \mathrm{~s}$ \\
\hline $\operatorname{RaQL}(T=50)$ & $0.537 \mathrm{~s}$ & $1.061 \mathrm{~s}$ & $6.135 \mathrm{~s}$ & $5.225 \mathrm{~s}$ \\
\hline $\operatorname{RaQL}(T=10)$ & $0.119 \mathrm{~s}$ & $0.302 \mathrm{~s}$ & $0.370 \mathrm{~s}$ & $1.329 \mathrm{~s}$ \\
\hline $\operatorname{RaQL}(T=5)$ & $0.062 \mathrm{~s}$ & $0.320 \mathrm{~s}$ & $0.286 \mathrm{~s}$ & $0.804 \mathrm{~s}$ \\
\hline $\operatorname{RaQL}(T=2)$ & $0.033 \mathrm{~s}$ & $0.169 \mathrm{~s}$ & $0.301 \mathrm{~s}$ & $0.350 \mathrm{~s}$ \\
\hline RaQL $(T=1)$ & $0.022 \mathrm{~s}$ & $0.064 \mathrm{~s}$ & $0.143 \mathrm{~s}$ & $0.529 \mathrm{~s}$ \\
\hline Standard $Q$-learning & $0.027 \mathrm{~s}$ & $0.125 \mathrm{~s}$ & $0.169 \mathrm{~s}$ & $0.374 \mathrm{~s}$ \\
\hline
\end{tabular}

Table 2: Computation Time 


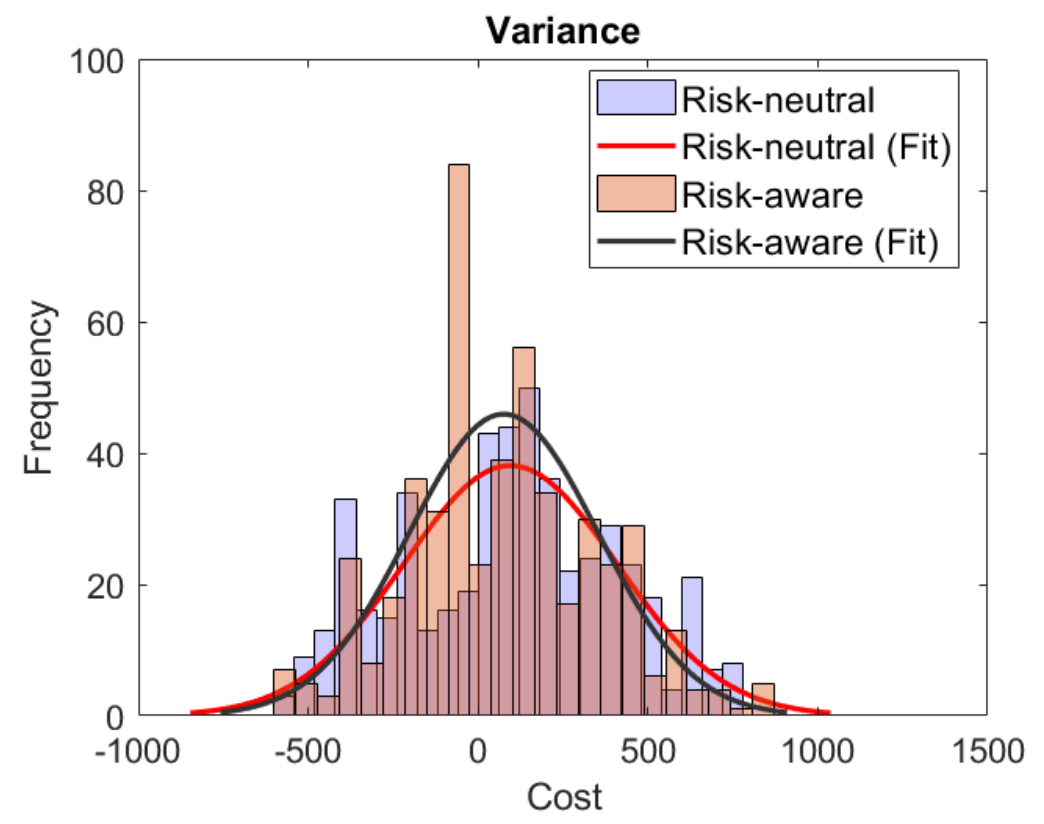

Figure 2: Policy Comparison

\subsection{Experiment II: RaQL vs. RsQL}

In this experiment, we compare the performance of RaQL with risk-sensitive $Q$-learning (RsQL) as proposed in [66]. We use the entropic risk measure (constructed from utility-based shortfall) as in [27, 29] to compare RaQL and RsQL. An entropic risk measure can be constructed from the utility function $u(x)=1-\exp (-\lambda x), \lambda>0$ for $x \in \mathbb{R}$ in OCE from Example 3.3 We set $\lambda=0.01$, the number of outer iterations to be $N=1 \times 10^{5}$, and the number of inner iterations to be $T=10$ for RaQL. The total number of iterations for RsQL is $1 \times 10^{5}$. The other settings remain the same as in Experiment I. Under these settings, RsQL terminates after 4.559s in expectation and RaQL uses 4.521s in expectation, to complete the first $1 \times 10^{4}$ iterations. Figure 2 shows that RaQL converges faster than RsQL. The convergence rate has also has lower standard deviation as shown by the error bars. We conjecture that the inner-outer loop structure of RaQL estimates the risk and updates the $Q$-values independently, which helps to reduce the bias in iterative $Q$-learning. In contrast, in RsQL, the risk estimation and $Q$-value updates are conducted simultaneously which may result in higher bias.

\subsection{Experiment III: SASP vs. Stochastic subgradient descent}

In this experiment, we compare RaQL with SASP and stochastic subgradient descent for risk estimation procedure to show that SASP has more accurate risk estimation. In particular, for stochastic subgradient descent we cut the moving average step (4.8), and change step 4.10] into

$$
\begin{aligned}
\left(y_{t+1}^{n}\left(s_{t}^{n}, a^{n}\right), z_{t+1}^{n}\left(s_{t}^{n}, a^{n}\right)\right)= & \Pi_{\mathcal{Y} \times \mathcal{Z}}\left\{\left(y_{t}^{n}\left(s_{t}^{n}, a^{n}\right), z_{t}^{n}\left(s_{t}^{n}, a^{n}\right)\right)\right. \\
& \left.-\lambda_{t, \alpha} \psi\left(v^{n-1}\left(s_{t+1}^{n}\right), y_{t}^{n}\left(s_{t}^{n}, a^{n}\right), z_{t}^{n}\left(s_{t}^{n}, a^{n}\right)\right)\right\},
\end{aligned}
$$

where the subgradient estimation of the current iteration is combined with computation of the saddle-point $\left(y_{t}^{n}\left(s_{t}^{n}, a^{n}\right), z_{t}^{n}\left(s_{t}^{n}, a^{n}\right)\right.$ (in SASP, the moving average of historical estimations $\left(y^{n, t}\left(s_{t}^{n}, a^{n}\right), z^{n, t}\left(s_{t}^{n}, a^{n}\right)\right)$ is used for this purpose). In this experiment, we set the number of outer iterations to be $N=3000$ and the number of inner iterations to be $T=100$, we take a linear learning rate $k=1$, and we set the step-size for risk estimation to be $\lambda_{t, \alpha}=C t^{-\alpha}$ with $\alpha=1 / 2$. We compare the two procedures for a functionally coherent risk measure (see Example 3.5. Figure 3 suggests that RaQL running on SASP has a lower relative error compared to the modified algorithm which uses stochastic subgradient descent, especially when the underlying risk measure is non-smooth and degenerate. 


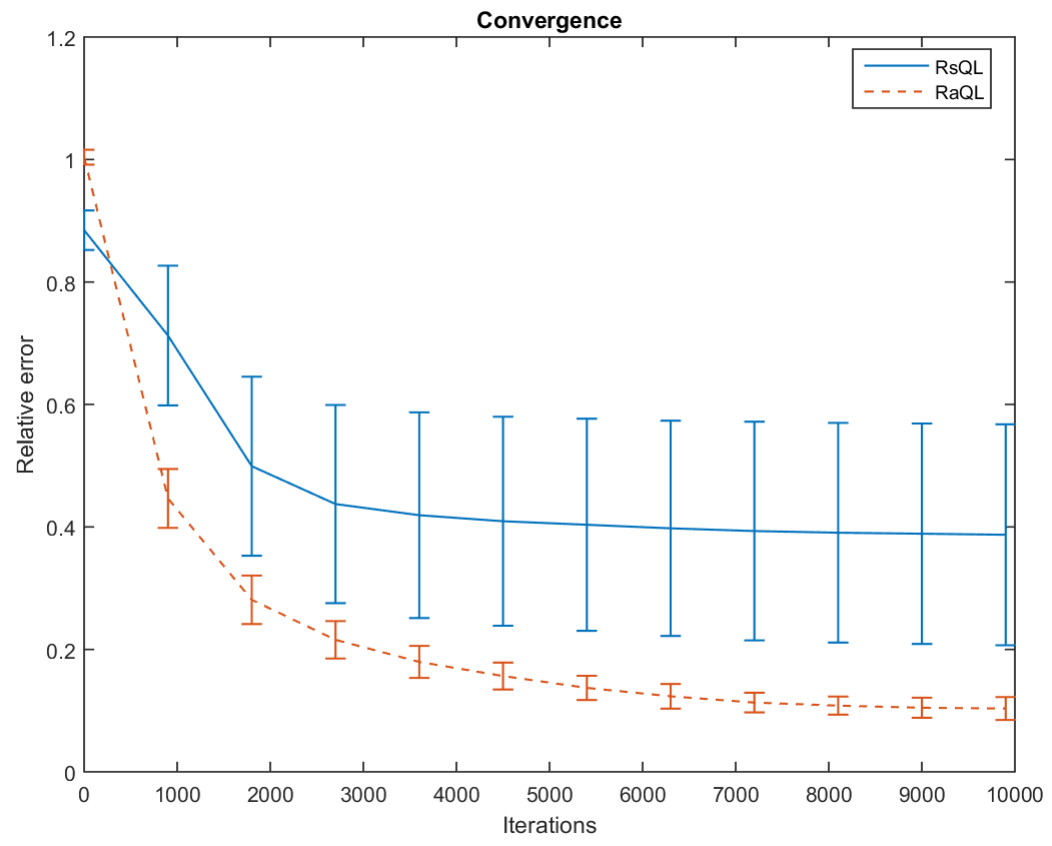

Figure 3: Numerical Experiment Result II

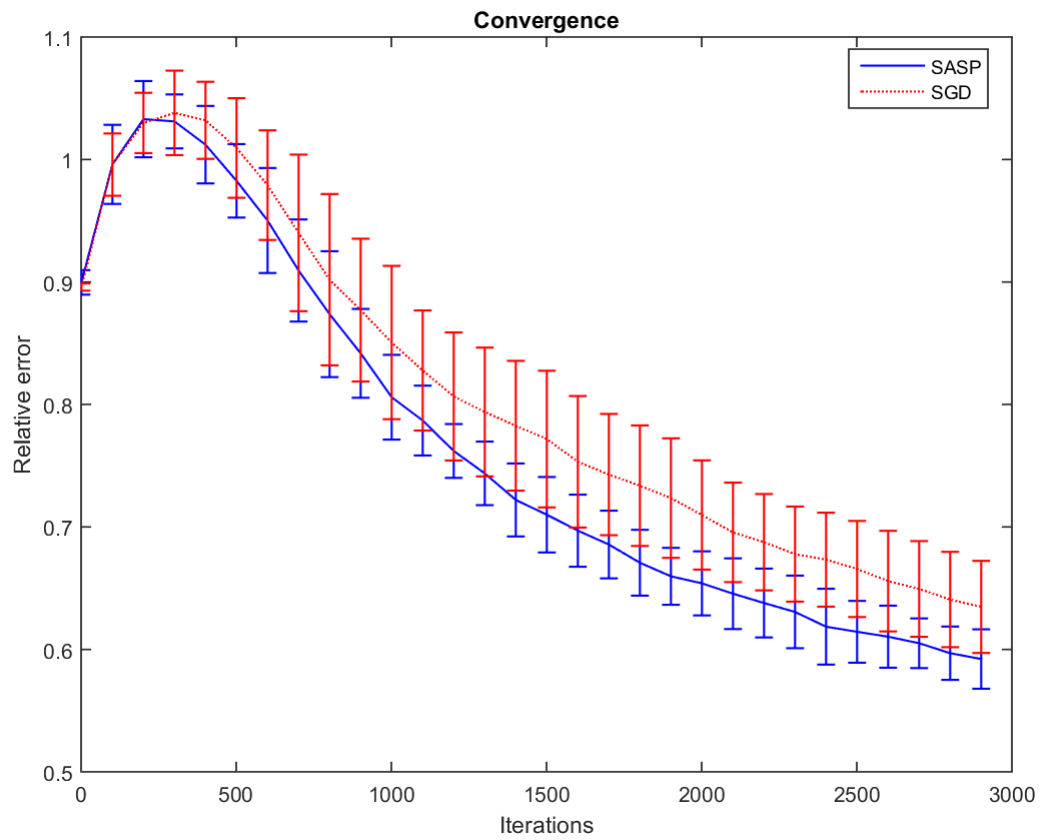

Figure 4: Numerical Experiment Result III 


\section{Conclusion}

We developed a new simulation-based algorithm for finite state/action, infinite-horizon, risk-aware Markov decision process, called Risk-aware $Q$-learning (RaQL). This algorithm can be used to solve many real life riskaware dynamic optimization problems in areas such as robotics, sequential online auctions, and infrastructure protection. We demonstrate that many commonly investigated risk measures (e.g. conditional value-at-risk, optimized certainty equivalent, absolute semi-deviation, and functionally coherent risk measures) fit into our framework. We analyze RaQL and establish both its almost sure convergence as well as its convergence rate $\left(\Omega\left(\left(\ln (1 / \delta \epsilon) / \epsilon^{2}\right)^{1 / k}+(\ln (1 / \epsilon))^{1 /(1-k)}\right)\right.$ with probability $1-\delta$, where $\epsilon>0$ is the error tolerance and $k \in(1 / 2,1]$ is the learning rate). For the case of a linear learning rate, we get an explicit convergence rate $(\Omega(1 / \epsilon))$ in expectation. Our experiments confirm the almost sure convergence of RaQL, and also show that RaQL has a convergence rate comparable to classical $Q$-learning in terms of the required number of outer loops. Additionally, our experiments illustrate the computational advantages of RaQL compared with some alternative methods for solving risk-aware MDPs.

In future research, we will explore new methods for speeding up the risk estimation subroutine to reduce the overall computational complexity, and we will also extend RaQL to handle continuous state and action spaces by incorporating $Q$-value function approximation techniques.

\section{Appendix}

Proof of Theorem 3.2. Let $P$ denote the probability distribution of $X$ and construct $\left\{h_{z}\right\}_{z \in \mathcal{Z}}$ satisfying conditions (i)-(iv) in the statement of the theorem. The stochastic saddle-point formulation in 3.2 is then

$$
\rho(X)=\min _{y \in\left[\eta_{\min }, \eta_{\max }\right]} \max _{z \in \mathcal{Z}}\left\{y+\mathbb{E}_{P}\left[h_{z}(X, y)\right]\right\} .
$$

Since $h_{z}$ is $P$-square summable for every $y \in \mathcal{Y}$ and $z \in \mathcal{Z}$, the corresponding function $G(3.2$ satisfies Assumption 3.1(i). Concavity of $h_{z}$ in $z \in \mathcal{Z}$ leads to this function $G$ satisfying Assumption 3.1(iii). Lipschitz continuity of $h_{z}$ with modulus $K_{G}-1$ implies that this function $G$ satisfies Assumption 3.1 (ii). The condition that the subgradients of $h_{z}(X-y)$ on $z \in \mathcal{Z}$ and $y \in \mathcal{Y}$ are Borel measurable and uniformly bounded, for any $X \in \mathcal{L}$, implies that this $G$ satisfies Assumption 3.1 (iv).

Next, we prove that formulation $(7.1)$ is a convex risk measure. Let $\phi(\cdot)$ denote a continuous and subdifferentiable $\phi$-divergence function for the distance between two probability distributions $P$ and $P^{\prime}$. We refer to [51. Table 5] for examples of $\phi$-divergence functions. Recall the Fenchel dual representation of convex risk measures,

$$
\rho(X)=\sup _{P^{\prime} \in \mathbb{P}}\left\{\mathbb{E}_{P^{\prime}}[X]-\mu\left(P^{\prime}\right)\right\},
$$

where $\mu$ is a convex function satisfying $\inf _{P^{\prime} \in \mathbb{P}} \mu\left(P^{\prime}\right)=0$, and $\mathbb{P}$ is the $\phi$-divergence risk envelope,

$$
\mathbb{P}:=\left\{P^{\prime}: P^{\prime} \geq 0,1^{\top} P^{\prime}=1, \int_{\Omega} \phi\left(\frac{d P^{\prime}}{d P}\right) d P \leq \beta\right\}
$$

consisting of all probability distributions with $\phi$-divergence from $P$ bounded by $\beta>0$. Let $\phi^{*}$ denote the convex conjugate of $\phi$ defined as $\phi^{*}(X):=\sup _{P^{\prime} \in \mathbb{P}}\left\{\mathbb{E}_{P^{\prime}}[X]-\phi\left(P^{\prime}\right)\right\}$. Based on the results for $\phi$-divergence risk envelopes constructed in 11, 12, 51, any convex risk measure 7.2 with corresponding set $\mathbb{P}$ can be reformulated as:

$$
\rho(X)=\inf _{b \geq 0, y \in \mathbb{R}}\left\{y+b \beta+b \mathbb{E}_{P}\left[\phi^{*}\left(\frac{X-y}{b}\right)\right]\right\} .
$$

Inspired by the minimax risk measure investigated in [59 60,62 64], we develop an extended variant for (7.3). Let $\phi_{z}$ denote a family of divergence functions parameterized by $z \in \mathcal{Z}$ that is concave in $z \in \mathcal{Z}$ for all fixed $X \in \mathcal{L}$, and let $\phi_{z}^{*}$ denote their corresponding convex conjugates. Define

$$
\mathbb{P}_{z}:=\left\{P^{\prime}: P^{\prime} \geq 0,1^{\top} P^{\prime}=1, \int_{\Omega} \phi_{z}\left(\frac{d P^{\prime}}{d P}\right) d P \leq \beta\right\}
$$


to be the set of probability distributions with bounded divergence with respect to $\phi_{z}, z \in \mathcal{Z}$, and set $\mathbb{P}_{\mathcal{Z}}=\bigcup_{z \in \mathcal{Z}} \mathbb{P}_{z}$. The equivalent form for 7.2 with the set $\mathbb{P}_{\mathcal{Z}}$ is now

$$
\rho(X)=\min _{b \geq 0, y \in \mathbb{R}} \max _{z \in \mathcal{Z}}\left\{y+b \beta+b \mathbb{E}_{P}\left[\phi_{z}^{*}\left(\frac{X-y}{b}\right)\right]\right\} .
$$

To complete the proof, given a constructed $\left\{h_{z}\right\}_{z \in \mathcal{Z}}$, and if we choose the $\phi$-divergence function with its convex conjugate $\phi^{*}$ satisfying

$$
\phi_{z}^{*}\left(\frac{X-y}{b}\right)=\frac{h_{z}(X, y)}{b}-\beta
$$

for any $y \in \mathbb{R}$ and $b \geq 0$, then the formulation 7.4 is equivalent to formulation (7.1). Thus formulation (7.1) is a convex risk measure.

Proof of Theorem 4.9 In this part, we detail the procedures to prove Theorem 4.9. As a remark, the natural logarithm term $e$ in 5.18 of Lemma 5.6 is specific to a polynomial learning rate, while for a linear learning rate we have a new relationship between $\tau_{m}$ and $\tau_{m+1}$. Thus, we must construct a different bound on $\left\{Y_{T}^{n, \tau_{m}}\right\}_{\tau_{m} \leq n \leq N}$, which is defined in Eq. (5.13). We first derive the convergence rate of process $Y_{T}^{n, \tau_{m}}(s, a)$ w.r.t. $T$. We prove the result by applying the same argument as in the proof of Lemma 5.6, and combining the arguments of [26, Lemma 22].

Lemma 7.1. Given any $m \geq 1$, assume that for all $n \geq \tau_{m}$ we have $Y_{T}^{n, \tau_{m}} \leq D_{m}$. Then for any $n \geq$ $(2+\Psi) \tau_{m}=\tau_{m+1}$, we have,

$$
Y_{T}^{n, \tau_{m}}(s, a) \leq D_{m}\left(K_{G}\left\{1-\gamma-\sqrt{\frac{C\left(\tau_{*}(T)\right)^{-\alpha}}{\kappa\left(1-C\left(\tau_{*}(T)\right)^{-\alpha} K_{\psi}^{(1)} \kappa\right)}}-K_{G} K_{S}\right\}+\frac{2}{2+\Psi} \beta_{T}\right),
$$

for all $(s, a) \in \mathbb{K}$, where $\beta_{T}$ is defined in (4.15) with $T$ satisfying conditions (4.16) and (4.17), $\Psi$ is any positive constant and $K_{s}$ is defined in (5.7).

The following Lemma enables the use of Azuma-Hoeffding inequality. Lemma 7.2 can be prove by applying the same argument in Lemma 5.7 , where we set $k=1$ because of linear learning rate.

Lemma 7.2. For any $n \geq \tau_{m}$ and $1 \leq l \leq n$ we have that $\left\{Z_{t}^{l, \tau_{m}}(s, a)\right\}_{l=1, \ldots, n}$ is a martingale difference sequence, which satisfies $\left|Z_{t}^{l, \tau_{m}}(s, a)-Z_{t}^{l-1, \tau_{m}}(s, a)\right| \leq \frac{V_{\max }}{(1-\varepsilon) \tau_{m}}$, for any $t \leq T$.

Lemma 7.3. Given $0<\delta<1$, we have (i)

$$
\mathbb{P}\left[\forall n \in\left[\tau_{m+1}, \tau_{m+2}\right]: Z_{T}^{n, \tau}(s, a) \leq \frac{\Psi}{2+\Psi} \beta_{T} D_{m}\right] \geq 1-\delta(1-\varepsilon),
$$

where $\tau_{m}=\Theta\left(\frac{V_{\max }^{2} \ln \left(V_{\max }|\mathbb{S}||\mathbb{A}| /\left[\Psi \delta \beta_{T} D_{m}(1-\varepsilon)\right]\right)}{\Psi^{2} \beta_{T} D_{m}^{2}(1-\varepsilon)^{2}}\right)$, and (ii)

$$
\mathbb{P}\left[\forall m \in\left[1, \frac{1}{1-\varepsilon}\right], \forall n \in\left[\tau_{m+1}, \tau_{m+2}\right]:\left|Z_{T}^{n, \tau}\right| \leq \tilde{\varepsilon}\right] \geq 1-\delta,
$$

where $\tau_{0}=\Theta\left(\frac{V_{\max }^{2} \ln \left(V_{\max }|\mathbb{S}||\mathbb{A}| /\left[\Psi \delta \beta_{T} \tilde{\varepsilon}(1-\varepsilon)\right]\right)}{\Psi^{2} \beta_{T} \tilde{\varepsilon}^{2}}\right)$.

Proof. Following the proofs of $\left[26\right.$. Lemma 37] and 26 . Lemma 38], we set $c_{i}=\Theta\left(\frac{V_{\max }}{(1-\varepsilon) \tau_{m}}\right)$, for any $n \geq \tau_{m+1}$, therefore we obtain

$$
\mathbb{P}\left[\left|Z_{T}^{n, \tau}\right| \geq \tilde{\varepsilon}\right] \leq 2 \exp \left(\frac{-\tilde{\varepsilon}^{2}}{2 \sum_{i=\tau_{m}+1, i \in N^{s, a}}^{n} c_{i}^{2}}\right) \leq 2 \exp \left(-c \frac{\tilde{\varepsilon}^{2} \tau_{m}(1+\Psi)}{V_{\max }^{2}}\right),
$$

for some constant $c>0$. Let us define the following variable

$$
\Xi^{n}(s, a)= \begin{cases}1, & \theta_{t, k}^{n} \neq 0 \\ 0 & \text { otherwise }\end{cases}
$$


where $k$ is fixed. Using the union bound and the fact in an interval of length $\frac{1+\Psi}{1-\varepsilon} \tau_{m}$, each state-action pair is visited at least $(1+\Psi) \tau_{m}$ times with certainty according to [26. Lemma 37], we get

$$
\begin{aligned}
\mathbb{P}\left[\forall n \in\left[\tau_{m+1}, \tau_{m+2}\right]:\left|Z_{T}^{n, \tau_{m}}\right| \geq \tilde{\varepsilon}\right] & \leq \mathbb{P}\left[\forall n \geq\left(\frac{1+\Psi}{1-\varepsilon}+1\right) \tau_{m}:\left|Z_{T}^{n, \tau_{m}}\right| \geq \tilde{\varepsilon}\right] \\
& \leq \sum_{n=((1+\Psi) /(1-\varepsilon)+1) \tau_{m}}^{\infty} \mathbb{P}\left[\left|Z_{T}^{n, \tau_{m}}(s, a)\right| \geq \tilde{\varepsilon}\right] \\
& \leq 2 \sum_{n=((1+\Psi) /(1-\varepsilon)+1) \tau_{m}}^{\infty} \Xi^{n}(s, a) \exp \left(-c \frac{\tau_{m}(1+\Psi) \tilde{\varepsilon}^{2}}{V_{\max }^{2}}\right) \\
& \leq 2 \exp \left(-c \frac{\left((1+\Psi) \tau_{m}\right) \tilde{\varepsilon}^{2}}{V_{\max }^{2}}\right) \sum_{n=0}^{\infty} \exp \left(-\frac{n \tilde{\varepsilon}^{2}}{2 V_{\max }^{2}}\right) \\
& =\frac{2 \exp \left(-c \frac{(1+\Psi) \tau_{m} \tilde{\varepsilon}^{2}}{V_{\max }^{2}}\right)}{1-\exp \left(-\frac{\tilde{\varepsilon}^{2}}{V_{\max }^{2}}\right)} \\
& =\Theta\left(\frac{\exp \left(-\frac{c^{\prime} \tau_{m} \tilde{\varepsilon}^{2}}{V_{\max }^{2}}\right)}{\tilde{\varepsilon}^{2}} V_{\max }^{2}\right)
\end{aligned}
$$

for some positive constant $c^{\prime}$. Controlling $\delta$ by setting

$$
\frac{\delta(1-\varepsilon)}{|\mathbb{S}||\mathbb{A}|}=\Theta\left(\frac{\exp \left(-\frac{c^{\prime} \tau_{m} \tilde{\varepsilon}^{2}}{V_{\max }^{2}}\right)}{\tilde{\varepsilon}^{2}} V_{\max }^{2}\right),
$$

which holds for $\tau_{m}=\Theta\left(\frac{V_{\max }^{2} \ln \left(V_{\max }|\mathbb{S}||\mathbb{A}| /\left(\delta D_{m}(1-\varepsilon)\right)\right.}{\beta_{T} D_{m}}\right)$, and $\tilde{\varepsilon}=\frac{\Psi}{2+\Psi} \beta_{T} D_{m}$ assures us that for every $t \geq \tau_{m+1}$ with probability at least $1-\delta(1-\varepsilon)$ the statement 7.5 holds at every state-action pair.

Theorem 4.9 follows from Lemma 7.3 , and the algebraic identity in the proof of $[26$. Theorem 5$]$ that

$$
a_{k+1}=a_{k}+\frac{(1+\Psi)}{1-\varepsilon} a_{k}=a_{0}\left(\frac{(1+\Psi)}{1-\varepsilon}+1\right)^{k} .
$$

The detailed proof follows the same procedures as the proof of Theorem 4.7

Proof of TheOrem 4.10 To start, we investigate the convergence rate of risk estimation step w.r.t. $t$ by stochastic approximation. We first refer to the convergence rate analysis of Algorithm 2 in [45]. As a measure of the quality of a candidate solution $(y, z) \in \mathcal{Y} \times \mathcal{Z}$, we use the duality gap $d(y, z)$ proposed by [45. Section 2.1.2]. Let $\bar{\Phi}(y)=\max _{z \in \mathcal{Z}} \mathbb{E}_{P}[G(X, y, z)]$, and $\underline{\Phi}(z)=\min _{y \in \mathcal{Y}} \mathbb{E}_{P}[G(X, y, z)]$, for any fixed $X \in \mathcal{L}$, and

$$
d(y, z):=\left[\bar{\Phi}(y)-\min _{y \in \mathcal{Y}} \bar{\Phi}(y)\right]+\left[\max _{z \in \mathcal{Z}} \underline{\Phi}(z)-\underline{\Phi}(z)\right]=\bar{\Phi}(y)-\underline{\Phi}(z) .
$$

The next theorem gives the convergence rate of SASP.

Theorem 7.4. [45, Theorem 1] Suppose Assumption 3.1 holds, set the step-size for all t as $\lambda_{t, \alpha}=C t^{-\alpha}, \alpha \in$ $(0,1]$, then for every $t>1$, we have

$$
\begin{aligned}
d\left(y^{t}, z^{t}\right) \leq & {\left[K_{\mathcal{Y}} H_{\mathcal{Y}}^{-1}+K_{\mathcal{Z}} H_{\mathcal{Z}}^{-1}\right] \frac{t^{\alpha}}{C\left(t-\tau_{*}(t)+1\right)}+\frac{\left(K_{\mathcal{Y}}+K_{\mathcal{Z}}\right) L}{\sqrt{t-\tau_{*}(t)+1}} } \\
& +C\left(K_{\mathcal{Y}}+K_{\mathcal{Z}}\right)^{2} L^{2}\left[H_{\mathcal{Y}} K_{\mathcal{Y}}+H_{\mathcal{Z}} K_{\mathcal{Z}}\right] \tau_{*}^{-\alpha}(t) .
\end{aligned}
$$


Lemma 7.5. Suppose Assumption 4.3 holds, for all $(s, a) \in \mathbb{K}, v \in \mathcal{V}$ and for every $t>1$ and $n \leq N$, we have the upper bound

$$
\begin{aligned}
& \mathbb{E}_{s^{\prime} \sim P(\cdot \mid s, a)}\left[\left|G\left(v\left(s^{\prime}\right), y^{n, t}(s, a), z^{n, t}(s, a)\right)-G\left(v\left(s^{\prime}\right), y^{n, *}(s, a), z^{n, *}(s, a)\right)\right|\right] \\
\leq & {\left[K_{\mathcal{Y}} H_{\mathcal{Y}}^{-1}+K_{\mathcal{Z}} H_{\mathcal{Z}}^{-1}\right] \frac{t^{\alpha}}{C\left(t-\tau_{*}(t)+1\right)}+\frac{\left(K_{\mathcal{Y}}+K_{\mathcal{Z}}\right) L}{\sqrt{t-\tau_{*}(t)+1}} } \\
& +C\left(K_{\mathcal{Y}}+K_{\mathcal{Z}}\right)^{2} L^{2}\left[H_{\mathcal{Y}} K_{\mathcal{Y}}+H_{\mathcal{Z}} K_{\mathcal{Z}}\right] \tau_{*}^{-\alpha}(t) .
\end{aligned}
$$

Proof. By the triangle inequality, we know that for all $(s, a) \in \mathbb{K}, v \in \mathcal{V}$ and for every $t>1$ and $n \leq N$,

$$
\begin{aligned}
& \mathbb{E}_{s^{\prime} \sim P(\cdot \mid s, a)}\left[\left|G\left(v\left(s^{\prime}\right), y^{n, t}(s, a), z^{n, t}(s, a)\right)-G\left(v\left(s^{\prime}\right), y^{n, *}(s, a), z^{n, *}(s, a)\right)\right|\right] \\
\leq & \max _{z \in \mathcal{Z}} \mathbb{E}_{s^{\prime} \sim P(\cdot \mid s, a)}\left[G\left(v\left(s^{\prime}\right), y^{n, t}(s, a), z^{n, t}(s, a)\right)\right] \\
& -\min _{y \in \mathcal{Y}} \mathbb{E}_{s^{\prime} \sim P(\cdot \mid s, a)}\left[G\left(v\left(s^{\prime}\right), y^{n, t}(s, a), z^{n, t}(s, a)\right)\right] .
\end{aligned}
$$

From Theorem 7.4, we know that

$$
\begin{aligned}
& \max _{z \in \mathcal{Z}} \mathbb{E}_{s^{\prime} \sim P(\cdot \mid s, a)}\left[G\left(v\left(s^{\prime}\right), y^{n, t}(s, a), z^{n, t}(s, a)\right)\right]-\min _{y \in \mathcal{Y}} \mathbb{E}_{s^{\prime} \sim P(\cdot \mid s, a)}\left[G\left(v\left(s^{\prime}\right), y^{n, t}(s, a), z^{n, t}(s, a)\right)\right] \\
\leq & {\left[K_{\mathcal{Y}} H_{\mathcal{Y}}^{-1}+K_{\mathcal{Z}} H_{\mathcal{Z}}^{-1}\right] \frac{t^{\alpha}}{C\left(t-\tau_{*}(t)+1\right)}+\frac{\left(K_{\mathcal{Y}}+K_{\mathcal{Z}}\right) L}{\sqrt{t-\tau_{*}(t)+1}} } \\
& +C\left(K_{\mathcal{Y}}+K_{\mathcal{Z}}\right)^{2} L^{2}\left[H_{\mathcal{Y}} K_{\mathcal{Y}}+H_{\mathcal{Z}} K_{\mathcal{Z}}\right] \tau_{*}^{-\alpha}(t) .
\end{aligned}
$$

Thus we obtain the desired result.

The next lemma bounds $\mathbb{E}\left[\left\|Q_{t}^{n}-Q^{*}\right\|_{2}^{2} \mid \mathcal{G}_{t+1}^{n-1}\right]$ w.r.t. $n$, for any $t \leq T$. For simplicity, we use function $f(t)$ to denote the right hand side of 7.7 .

Lemma 7.6. The sequence $Q_{t}^{n}$, generated by Algorithm 1 satisfies, for any $\kappa_{0}, \kappa>0$,

$$
\begin{aligned}
\mathbb{E}\left[\left\|Q_{t}^{n}-Q^{*}\right\|_{2}^{2} \mid \mathcal{G}_{t+1}^{n-1}\right] & \leq\left[1-\frac{1}{n^{k}}\left(2 \varepsilon-\gamma K_{G} \kappa_{0}-\gamma K_{G} \kappa-\gamma K_{G} / \kappa_{0}-K_{S} K_{G}^{2} / \kappa\right)\right] \mathbb{E}\left[\left\|Q_{T}^{n-1}-Q^{*}\right\|_{2}^{2} \mid \mathcal{G}_{t+1}^{n-1}\right] \\
& +\frac{\left(C_{G}+(\gamma f(t))^{2}\right)}{n^{2 k}},
\end{aligned}
$$

where $C_{G}$ bounds the term

$$
\left\{1+\frac{C}{\kappa\left(1-C K_{\psi}^{(1)} \kappa\right)}+\left[K_{G}\left(\gamma+K_{S} K_{G}\right)\right]^{2}\right\}\left\|Q_{T}^{n-1}-Q^{*}\right\|_{2}^{2} \leq C_{G} .
$$

Proof. Let us recall that the update of Step 3 in Algorithm 1 is equivalent to

$$
\left.Q_{t}^{n}=Q_{T}^{n-1}-\theta_{k}^{n}\left[Q_{T}^{n-1}-Q^{*}+\xi_{t}^{n}+\epsilon_{t}^{n}+Q^{*}-H\left(v^{*}, y^{*}, z^{*}\right)\right)\right] .
$$

Expanding, we have

$$
\begin{aligned}
\left\|Q_{t}^{n}-Q^{*}\right\|_{2}^{2} \leq & \left\|Q_{T}^{n-1}-Q^{*}\right\|_{2}^{2}+\left\|\theta_{k}^{n}\left[Q_{T}^{n-1}-Q^{*}+\epsilon_{t}^{n}+\xi_{t}^{n}\right]\right\|_{2}^{2} \\
& -2\left(Q_{T}^{n-1}-Q^{*}\right)^{\top} \theta_{k}^{n}\left[Q_{T}^{n-1}-Q^{*}+\epsilon_{t}^{n}+\xi_{t}^{n}\right] .
\end{aligned}
$$

We focus on the cross term $-2\left(Q_{T}^{n-1}-Q^{*}\right)^{\top} \theta_{k}^{n}\left[Q_{T}^{n-1}-Q^{*}+\epsilon_{t}^{n}+\xi_{t}^{n}\right]$. First, by the $\varepsilon$-greedy exploration policy, notice that

$$
\mathbb{E}_{p}\left[\left(Q_{T}^{n-1}-Q^{*}\right)^{\top} \theta_{k}^{n}\left(Q_{T}^{n-1}-Q^{*}\right) \mid \mathcal{G}_{t+1}^{n-1}\right] \geq \frac{\varepsilon}{n^{k}}\left\|Q_{T}^{n-1}-Q^{*}\right\|_{2}^{2},
$$


and by the definition of $\epsilon_{t}^{n}$, we have by Lemma 7.5, that

$$
\begin{aligned}
\mathbb{E}\left[\epsilon_{t}^{n} \mid \mathcal{G}_{t+1}^{n-1}\right] & =\mathbb{E}\left[H\left(v^{n-1}, y^{n, *}, z^{n, *}\right)-H\left(v^{n-1}, y^{n, t}, z^{n, t}\right) \mid \mathcal{G}_{t+1}^{n-1}\right] \\
& \leq \gamma \mathbb{E}_{s^{\prime} \sim P(\cdot \mid s, a)}\left[\left|G\left(v^{n-1}\left(s^{\prime}\right), y^{n, t}(s, a), z^{n, t}(s, a)\right)-G\left(v^{n-1}\left(s^{\prime}\right), y^{n, *}(s, a), z^{n, *}(s, a)\right)\right|\right] \\
& \leq \gamma f(t),
\end{aligned}
$$

and

$$
\mathbb{E}\left[-\left(Q_{T}^{n-1}-Q^{*}\right)^{\top} \theta_{k}^{n} \epsilon_{t}^{n} \mid \mathcal{G}_{t+1}^{n-1}\right] \leq \frac{\gamma f(t)}{n^{k}}\left\|Q_{T}^{n-1}-Q^{*}\right\|_{2} .
$$

Applying the algebraic identity $2 a b \leq a^{2} \kappa+b^{2} / \kappa$ for all $\kappa>0$, we see that

$$
\mathbb{E}\left[-2\left(Q_{T}^{n-1}-Q^{*}\right)^{\top} \theta_{k}^{n} \epsilon_{t}^{n} \mid \mathcal{G}_{t+1}^{n-1}\right] \leq \kappa\left\|Q_{T}^{n-1}-Q^{*}\right\|_{2}^{2}+\frac{(\gamma f(t))^{2}}{n^{2 k} \kappa} .
$$

We can see that

$$
\begin{aligned}
\mathbb{E}\left[-\left(Q_{T}^{n-1}-Q^{*}\right)^{\top} \theta_{k}^{n} \xi_{t}^{n} \mid \mathcal{G}_{t+1}^{n-1}\right] \leq & \frac{\gamma^{2}}{n^{k}} K_{G}\left[\left\|Q_{T}^{n-1}-Q^{*}\right\|_{2}\left\|Q_{T}^{n-1}-Q^{*}\right\|_{2}\right. \\
& \left.+\gamma\left\|Q_{T}^{n-1}-Q^{*}\right\|_{2}\left(\left\|\left(y^{n, *}, z^{n, *}\right)-\left(y^{*}, z^{*}\right)\right\|_{2}\right)\right] .
\end{aligned}
$$

Again applying the algebraic identity $2 a b \leq a^{2} \kappa+b^{2} / \kappa$ for all $\kappa>0$, we see that for any constants $\kappa_{0}, \kappa>0$,

$$
\begin{aligned}
\mathbb{E}\left[-2\left(Q_{T}^{n-1}-Q^{*}\right)^{\top} \theta_{k}^{n} \xi_{t}^{n} \mid \mathcal{G}_{t+1}^{n-1}\right] \leq & \frac{\gamma}{n^{k}} K_{G}\left[\left(\kappa_{0}+\kappa\right)\left\|Q_{T}^{n-1}-Q^{*}\right\|_{2}^{2}\right] \\
& +\frac{\gamma K_{G}}{n^{k} \kappa_{0}}\left\|Q_{T}^{n-1}-Q^{*}\right\|_{2}^{2}+\frac{K_{G}}{n^{k} \kappa}\left(\left\|\left(y^{n, *}, z^{n, *}\right)-\left(y^{*}, z^{*}\right)\right\|_{2}^{2}\right) \\
\leq & \frac{\gamma}{n^{k}} K_{G}\left[\left(\kappa_{0}+\kappa\right)\left\|Q_{T}^{n-1}-Q^{*}\right\|_{2}^{2}\right] \\
& +\frac{\gamma K_{G}}{n^{k} \kappa_{0}}\left\|Q_{T}^{n-1}-Q^{*}\right\|_{2}^{2}+\frac{K_{S} K_{G}^{2}}{n^{k} \kappa}\left\|Q_{T}^{n-1}-Q^{*}\right\|_{2}^{2} .
\end{aligned}
$$

Finally, there exists $\kappa>0$ such that

$$
\begin{aligned}
& \left\|\theta_{k}^{n}\left[Q_{T}^{n-1}-Q^{*}+\epsilon_{t}^{n}+\xi_{t}^{n}\right]\right\|_{2}^{2} \\
\leq & \frac{1}{n^{2 k}}\left\{1+\frac{C}{\kappa\left(1-C K_{\psi}^{(1)} \kappa\right)}+\left[K_{G}\left(\gamma+K_{S} K_{G}\right)\right]^{2}\right\}\left\|Q_{T}^{n-1}-Q^{*}\right\|_{2}^{2},
\end{aligned}
$$

where the above inequality holds based on (5.10) and 5.11). For simplicity, let $C_{G}$ bound the term

$$
\left\{1+\frac{C}{\kappa\left(1-C K_{\psi}^{(1)} \kappa\right)}+\left[K_{G}\left(\gamma+K_{S} K_{G}\right)\right]^{2}\right\}\left\|Q_{T}^{n-1}-Q^{*}\right\|_{2}^{2} .
$$

The statement of the lemma follows by taking expectations of inequalities $77.10,77.11,77.12$ and 7.13 , and using the inequality (7.14), and combining.

The convergence rate in expectation with a linear learning rate is based on the following result from [25], which is useful for analyzing a specific type of sequence that often arises in recursive algorithms.

Lemma 7.7. [25] Consider a sequence $\left\{a^{n}\right\}$. Suppose for some $b>1$ and every $n \geq 1$ that $a^{n} \leq$ $\left(1-\frac{b}{n}\right) a^{n-1}+\frac{c}{n^{2}}$. Then, if $d \geq \max \left\{\frac{c}{b-1}, a^{0}\right\}$, it follows that $a^{n} \leq \frac{d}{n}$ for $n \geq 1$.

Based on the results of Lemma 7.6 , for linear learning rate $k=1$, we let $a^{n}=\mathbb{E}\left[\left\|Q_{t}^{n}-Q^{*}\right\|_{2}^{2} \mid \mathcal{G}_{t+1}^{n}\right]$ for any $t \leq T$, and choose

$$
d \geq \max \left\{\frac{\left(C_{G}+(\gamma f(t))^{2}\right)}{\left(2 \varepsilon-\gamma K_{G} \kappa_{0}-\gamma K_{G} \kappa-\gamma K_{G} / \kappa_{0}-K_{S} K_{G}^{2} / \kappa\right)-1}, C_{\max }^{2}|\mathbb{S}||\mathbb{A}|\right\},
$$


in Lemma 7.7, where we also have $a^{0}=\mathbb{E}\left[\left\|Q_{t}^{1}-Q^{*}\right\|_{2}^{2} \mid \mathcal{G}_{t+1}^{0}\right] \leq C_{\max }^{2}|\mathbb{S}||\mathbb{A}|$. By setting $\kappa=\kappa_{0}=\varepsilon$, given a small positive constant $\tilde{\varepsilon}>0$, we have the sample complexity

$$
N=\Omega\left(\frac{\max \left\{\left(C_{G}+(\gamma f(t))^{2}\right) \varepsilon /\left(\left(2-2 \gamma K_{G}\right) \varepsilon^{2}-K_{G}\left(\gamma-K_{S} K_{G}\right)-\varepsilon\right), C_{\max }^{2}|\mathbb{S}||\mathbb{A}|\right\}}{\tilde{\varepsilon}}\right),
$$

such that $\mathbb{E}\left[\left\|Q_{t}^{N}-Q^{*}\right\|_{2}^{2} \mid \mathcal{G}_{t+1}^{N-1}\right] \leq \tilde{\varepsilon}$, for any $t \leq T$. We have thus proved the desired result summarized in Theorem 4.10

\section{References}

[1] Sherief Abdallah and Michael Kaisers. Addressing environment non-stationarity by repeating q-learning updates. The Journal of Machine Learning Research, 17(1):1582-1612, 2016.

[2] Carlo Acerbi. Spectral measures of risk: a coherent representation of subjective risk aversion. Journal of Banking \& Finance, 26(7):1505-1518, 2002.

[3] Mohammad Abu Alsheikh, Dinh Thai Hoang, Dusit Niyato, Hwee-Pink Tan, and Shaowei Lin. Markov decision processes with applications in wireless sensor networks: A survey. IEEE Communications Surveys \& Tutorials, 17(3):1239-1267, 2015.

[4] Philippe Artzner, Freddy Delbaen, Jean-Marc Eber, and David Heath. Coherent measures of risk. Mathematical finance, 9(3):203-228, 1999.

[5] Hédy Attouch and Gerald Beer. On the convergence of subdifferentials of convex functions. Archiv der Mathematik, 60(4):389-400, 1993.

[6] Kazuoki Azuma. Weighted sums of certain dependent random variables. Tohoku Mathematical Journal, Second Series, 19(3):357-367, 1967.

[7] Olivier Bardou, Noufel Frikha, and Gilles Pages. Computing var and cvar using stochastic approximation and adaptive unconstrained importance sampling. Monte Carlo Methods and Applications, 15(3):173$210,2009$.

[8] Nicole Bäuerle and Jonathan Ott. Markov decision processes with average-value-at-risk criteria. Mathematical Methods of Operations Research, 74(3):361-379, 2011.

[9] Nicole Bäuerle and Ulrich Rieder. More risk-sensitive markov decision processes. Mathematics of Operations Research, 39(1):105-120, 2013.

[10] Richard Bellman. A markovian decision process. Technical report, DTIC Document, 1957.

[11] Aharon Ben-Tal, Dick Den Hertog, Anja De Waegenaere, Bertrand Melenberg, and Gijs Rennen. Robust solutions of optimization problems affected by uncertain probabilities. Management Science, 59(2):341$357,2013$.

[12] Aharon Ben-Tal, Dick Den Hertog, and Jean-Philippe Vial. Deriving robust counterparts of nonlinear uncertain inequalities. Mathematical Programming, 149(1-2):265-299, 2015.

[13] Aharon Ben-Tal and Marc Teboulle. An old-new concept of convex risk measures: The optimized certainty equivalent. Mathematical Finance, 17(3):449-476, 2007.

[14] Dimitri P Bertsekas and John N Tsitsiklis. Neuro-dynamic programming. Athena Scientific Belmont, MA, 1996.

[15] Dimitris Bertsimas and David B Brown. Constructing uncertainty sets for robust linear optimization. Operations research, 57(6):1483-1495, 2009. 
[16] Vivek Borkar and Rahul Jain. Risk-constrained markov decision processes. Automatic Control, IEEE Transactions on, 59(9):2574-2579, 2014.

[17] Vivek S Borkar. Q-learning for risk-sensitive control. Mathematics of operations research, 27(2):294-311, 2002.

[18] Vivek S Borkar et al. Stochastic approximation. Cambridge Books, 2008.

[19] Vivek S Borkar and Sean P Meyn. The ode method for convergence of stochastic approximation and reinforcement learning. SIAM Journal on Control and Optimization, 38(2):447-469, 2000.

[20] Stephen Boyd and Lieven Vandenberghe. Convex Optimization. Cambridge University Press, 2004.

[21] Leo Breiman. Probability, volume 7 of classics in applied mathematics. Society for Industrial and Applied Mathematics (SIAM), Philadelphia, PA, 1992.

[22] David B Brown, Enrico De Giorgi, and Melvyn Sim. Aspirational preferences and their representation by risk measures. Management Science, 58(11):2095-2113, 2012.

[23] Peter Carbonetto, Mark Schmidt, and Nando D Freitas. An interior-point stochastic approximation method and an 11-regularized delta rule. In Advances in neural information processing systems, pages 233-240, 2009.

[24] Yinlam Chow, Mohammad Ghavamzadeh, Lucas Janson, and Marco Pavone. Risk-constrained reinforcement learning with percentile risk criteria. Journal of Machine Learning Research, 18(167):1-51, 2018.

[25] Kai Lai Chung. On a stochastic approximation method. The Annals of Mathematical Statistics, pages 463-483, 1954.

[26] Eyal Even-Dar and Yishay Mansour. Learning rates for q-learning. The Journal of Machine Learning Research, 5:1-25, 2004.

[27] Hans Föllmer and Thomas Knispel. Entropic risk measures: Coherence vs. convexity, model ambiguity and robust large deviations. Stochastics and Dynamics, 11(02n03):333-351, 2011.

[28] Hans Föllmer and Alexander Schied. Convex measures of risk and trading constraints. Finance and stochastics, 6(4):429-447, 2002.

[29] Hans Föllmer and Alexander Schied. Stochastic finance: an introduction in discrete time. Walter de Gruyter, 2011.

[30] William B. Haskell and Rahul Jain. Stochastic dominance-constrained markov decision processes. SIAM J. Control Optim., 51(1):273-303, 2013.

[31] William B Haskell and Rahul Jain. A convex analytic approach to risk-aware markov decision processes. SIAM Journal on Control and Optimization, 53(3):1569-1598, 2015.

[32] Hado V Hasselt. Double q-learning. In Advances in Neural Information Processing Systems, pages 2613-2621, 2010.

[33] Junling Hu and Michael P Wellman. Nash q-learning for general-sum stochastic games. Journal of machine learning research, 4(Nov):1039-1069, 2003.

[34] Qiying $\mathrm{Hu}$ and Wuyi Yue. Markov decision processes with their applications, volume 14. Springer Science \& Business Media, 2007.

[35] Tommi Jaakkola, Michael I Jordan, and Satinder P Singh. On the convergence of stochastic iterative dynamic programming algorithms. Neural computation, 6(6):1185-1201, 1994.

[36] Daniel R Jiang and Warren B Powell. Risk-averse approximate dynamic programming with quantilebased risk measures. Mathematics of Operations Research, 43(2):554-579, 2017. 
[37] Simon Keizer, Mary Ellen Foster, Oliver Lemon, Andre Gaschler, and Manuel Giuliani. Training and evaluation of an mdp model for social multi-user human-robot interaction. In Proceedings of the SIGDIAL 2013 Conference, pages 223-232, 2013.

[38] Pavlo Krokhmal, Jonas Palmquist, and Stanislav Uryasev. Portfolio optimization with conditional value-at-risk objective and constraints. Journal of risk, 4:43-68, 2002.

[39] Filip Krsmanovic, Curtis Spencer, Daniel Jurafsky, and Andrew Y Ng. Have we met? mdp based speaker id for robot dialogue. In Ninth International Conference on Spoken Language Processing, 2006.

[40] Harold J. Kushner and G.George Yin. Stochastic Approximation and Recursive Algorithms and Applications. Springer, 2003.

[41] Adam B Levy, Ren6 A Poliquin, and R Tyrrell Rockafellar. Stability of locally optimal solutions. SIAM Journal on Optimization, 10(2):580-604, 2000.

[42] Karthik Natarajan, Dessislava Pachamanova, and Melvyn Sim. Constructing risk measures from uncertainty sets. Operations research, 57(5):1129-1141, 2009.

[43] Angelia Nedić and Asuman Ozdaglar. Subgradient methods for saddle-point problems. Journal of optimization theory and applications, 142(1):205-228, 2009.

[44] A. Nemirovski, A. Juditsky, G. Lan, and A. Shapiro. Robust stochastic approximation approach to stochastic programming. Society of Industrial and Applied Mathematics Journal of Optimization, 19(4):1574-1609, 2009.

[45] Arkadi Nemirovski and Reuven Rubinstein. An efficient stochastic approximation algorithm for stochastic saddle point problems. Modeling Uncertainty, pages 156-184, 2005.

[46] Nilay Noyan and Gábor Rudolf. Optimization with multivariate conditional value-at-risk constraints. Operations Research, 61(4):990-1013, 2013.

[47] Nilay Noyan and GÃ bor Rudolf. Kusuoka representations of coherent risk measures in general probability spaces. Annals of Operations Research, 229(1):591, June 2015.

[48] Jonathan Theodor Ott. A Markov decision model for a surveillance application and risk-sensitive Markov decision processes. PhD thesis, Karlsruher Inst. für Technologie, Diss., 2010, 2010.

[49] Jean-Paul Penot. On the convergence of subdifferentials of convex functions. Nonlinear Analysis: Theory, Methods \& Applications, 21(2):87-101, 1993.

[50] Andy Philpott, Vitor de Matos, and Erlon Finardi. On solving multistage stochastic programs with coherent risk measures. Operations Research, 61(4):957-970, 2013.

[51] Krzysztof Postek, Dick den Hertog, and Bertrand Melenberg. Computationally tractable counterparts of distributionally robust constraints on risk measures. SIAM Review, 58(4):603-650, 2016.

[52] Warren B Powell. Approximate Dynamic Programming: Solving the curses of dimensionality, volume 703. John Wiley \& Sons, 2007.

[53] LA Prashanth. Policy gradients for cvar-constrained mdps. In International Conference on Algorithmic Learning Theory, pages 155-169. Springer, 2014.

[54] R. T. Rockafellar and R.J-B. Wets. Variational Analysis. Springer-Verlag, Berlin, 1998.

[55] R. Tyrrell Rockafellar. Convex Analysis. Princeton University Press, 1970.

[56] R Tyrrell Rockafellar and Stanislav Uryasev. Optimization of conditional value-at-risk. Journal of risk, $2: 21-42,2000$.

[57] Andrzej Ruszczyński. Risk-averse dynamic programming for markov decision processes. Mathematical programming, 125(2):235-261, 2010. 
[58] Andrzej Ruszczynski and Alexander Shapiro. Optimization of convex risk functions. Mathematics of operations research, 31(3):433-452, 2006.

[59] Alexander Shapiro. On duality theory of convex semi-infinite programming. Optimization, 54(6):535543,2005 .

[60] Alexander Shapiro. Minimax and risk averse multistage stochastic programming. European Journal of Operational Research, 219(3):719-726, 2012.

[61] Alexander Shapiro. On kusuoka representation of law invariant risk measures. Mathematics of Operations Research, 38(1):142-152, 2013.

[62] Alexander Shapiro and Shabbir Ahmed. On a class of minimax stochastic programs. SIAM Journal on Optimization, 14(4):1237-1249, 2004.

[63] Alexander Shapiro, Darinka Dentcheva, and Andrzej Ruszczyński. Lectures on Stochastic Programming Modeling and Theory. Society for Industrial and Applied Mathematics and the Mathematical Programming Society, 2009.

[64] Alexander Shapiro and Anton Kleywegt. Minimax analysis of stochastic problems. Optimization Methods and Software, 17(3):523-542, 2002.

[65] Yun Shen, Wilhelm Stannat, and Klaus Obermayer. Risk-sensitive markov control processes. SIAM Journal on Control and Optimization, 51(5):3652-3672, 2013.

[66] Yun Shen, Michael J Tobia, Tobias Sommer, and Klaus Obermayer. Risk-sensitive reinforcement learning. Neural computation, 26(7):1298-1328, 2014.

[67] Aviv Tamar, Yonatan Glassner, and Shie Mannor. Policy gradients beyond expectations: Conditional value-at-risk. arXiv preprint arXiv:1404.3862, 2014.

[68] Yasushi Terazono and Ayumu Matani. Continuity of optimal solution functions and their conditions on objective functions. SIAM Journal on Optimization, 25(4):2050-2060, 2015.

[69] John N Tsitsiklis. Asynchronous stochastic approximation and q-learning. Machine Learning, 16(3):185202, 1994.

[70] Hado Van Hasselt, Arthur Guez, and David Silver. Deep reinforcement learning with double q-learning. In $A A A I$, volume 2, page 5. Phoenix, AZ, 2016.

[71] Christopher JCH Watkins and Peter Dayan. Q-learning. Machine learning, 8(3-4):279-292, 1992. 